\title{
Aizoon Extract As a Novel Eco-friendly Corrosion Inhibitor For Stainless Steel430 in HCl Solution
}

\author{
Ahmed F. Molouk \\ Mansoura University \\ Mohamed F. Atia \\ Delta Technological University \\ Abd El-Aziz S. Fouda ( $\square$ asfouda@hotmail.com ) \\ Mansoura University
}

\section{Research Article}

Keywords: hydrochloric, electrochemical, potentiodynamic, Polarization

Posted Date: October 21st, 2021

DOI: https://doi.org/10.21203/rs.3.rs-908157/v1

License: (1) This work is licensed under a Creative Commons Attribution 4.0 International License. Read Full License 


\title{
Aizoon extract as a novel eco-friendly corrosion inhibitor for stainless steel430 in $\mathrm{HCl}$ solution
}

\begin{abstract}
Ahmed F. Molouk ${ }^{1}$, Mohamed F. Atia ${ }^{2}$, Abd El-Aziz S. Fouda*1
${ }^{1}$ Chemistry Department, Faculty of Science, Mansoura University, Mansoura-35516, Egypt, email: asfouda@hotmail.com, Tel:+2 050 2365730,Fax\# +2 0502202264

${ }^{2}$ Faculty of Technological Industry and Energy, Delta Technological University, Qewasnia, ElMenoufia, Egypt

Aizoon extract used as anti-corrosion for stainless steel430 (SS430) in 2M hydrochloric acid solution. The mass reduction, electrochemical impedance, and potentiodynamic polarization were carried out to demonstrate the performance of Aizoon extract as corrosion resistance for SS430. Polarization revealed that the Aizoon extract is mixed type inhibitor with superiority to inhibiting the cathodic reaction. The inhibition percentage reaches $93 \%$ at $300 \mathrm{ppm}$ extract. Adsorption of the extract on SS430 surface is regular with Langmuir adsorption model. Thermodynamic factors for adsorption and activation processes for SS430 dissolution were estimated and discussed. Furthermore, the SS430 surface is characterized using different techniques. The surface morphology of SS430 was tested utilizing several techniques. The experimental data were supported by the theoretical data.
\end{abstract}

Because of its vast range of uses in industry, such as automobile accessories, culinary utensils, cleaning equipment components, and industrial pipes, global SS production has increase rapidly in recent decades. Furthermore, the tanks used to transport nitric acid are frequently constructed of SS430. Because of the development of a thin passive coating of chromium ox-hydroxide that protects the alloy, stainless steel has a high corrosion resistance [1]. However, in an acidic medium as $\mathrm{HCl}$ the passive film formed over the SS surface will be disappeared as chromium reacts with chloride producing chromium chloride. The chromium will be gradually lost from SS leaving just iron [2-5]. Therefore, using corrosion extracts is a very beneficial strategy for protecting SS against corrosion where the extracts will be adsorbed on SS active sites and in turn protects it from a corrosive medium. Organic compounds are the most dominant extracts used in industry. Often, the chemical structure of efficient extracts should contain donor atoms as oxygen, sulfur, and nitrogen and/or double and triple bonds which assists the absorption of these organic compounds on metal surface through bonding the electron pair of the donor atoms with the active sites on metal surface. Therefore, the corrosive attack in an acidic medium will be retarded [6-14]. Despite the high efficiency of organic extracts, they have a lot of drawbacks due to the environmental problems associated with it even if they added by small concentration. Attention has shifted to naturally occurring substances because they are environmentally friendly. Plant extract covers this problem as its biodegradable, absence of hazardous compound and heavy metals, and safe for the environment. Numerous researches were carried out using extracts from plants as successful anticorrosion for iron and steel in acidic environment. Qiang et al investigated the inhibition influence of extract from Ginkgo leaves on X70 steel in $1 \mathrm{M} \mathrm{HCl}$ by conducting electrochemical 
measurements [15]. The inhibition efficiency exceeded $90 \%$ by adding $200 \mathrm{ppm}$ of the extract at all tested temperatures demonstrating an excellent inhibition capacity. In other research, the inhibition characteristics were investigated for the extract which obtained from Dendrocalamus brandisii leaves to protect cold rolled steel in trichloroacetic acid by measuring the loss in weight and electrochemical tests. The results showed that the extract is a good extract with maximum inhibition percentage more than 95\% [16]. A study was done by Suprapto et al on the performance of Acera Alba extract as anti-corrosion for SS304 in $\mathrm{HCl}$. The polarization study revealed that the extract acts as a mixed extract give inhibition up to 90\%. The adsorption on SS304 obeyed Frumkin isotherm [17]. Moreover, Gapsari et al. confirmed the extract action of an extract from bee wax propolis on the SS304 in $0.5 \mathrm{M}$ sulfuric acid. The results showed that the extract with concentration $2000 \mathrm{ppm}$ retarded the corrosion rate and the inhibition percentage reached 97.29\% [18]. Therefore, plant extracts are promising extracts for protecting metals and alloys from corrosion. The data gained from the various studies showed that plant extracts could serve as effective inhibitors for corrosion. In this work the Aizoon extract was selected to be investigated because of its constituent compounds are promising as corrosion extract [19] beside its availability in several regions and ease to obtain the extract. Plant extract, Aizoon, is a genus of flowering plants in the ice plant family of aizoaceae it is originating in the Mediterranean region, and widely cultivated in North Africa from the Atlantic Islands (Canaries) and Cape Verde eastward through northern Africa to Egypt and Sudan. On the other hand, there is a shortage in the research which study the protection of SS430 from corrosion in an acidic medium compared to other SS grades. Therefore, the aim of this work is to investigate the inhibition performance of the Aizoon extract on the corrosion of SS type 430 in $2 \mathrm{M}$ hydrochloric acid using mass reduction (MR), potentiodynamic polarization (PDP), and electrochemical impedance spectroscopy (EIS) test. In addition, the surface of SS430 was characterized by scanning electron microscopy (SEM), atomic force spectroscope (AFM) and Fourier transform infrared spectroscopy (FTIR) analyses.

\section{Experimental methods}

\section{Extraction of Aizoon plant}

Plant used in this work was collected from Sadat city desert, Egypt. Leaves of Aizoon were prewashed and then dried in air at room temperature away from direct light for several days. The dried leaves were coarsely crushed in a cylindrical crusher then sieved to obtain a homogeneous powder. The extract was obtained by soaking $200 \mathrm{~g}$ of the leaves' powder in $200 \mathrm{ml}$ methanol $70 \%$ (ADWIC, Nasr Pharma, Egypt) for $48 \mathrm{~h}$. The powder was removed by filtration to get a clear filtrate. Subsequently, the filtrate was vaporized under vacuum. The residue after evaporation was collected and stored blow $273 \mathrm{~K}$ in a glass flask stoppered with screw plastic lid. It was confirmed that, the Aizoon extract consist of eleven compounds were identified as: 5,7-dihydroxy chromone, pyrogallol, kaempferol, protocatechuic acid, vallinic acid, thymine, caffeic acid, quercetin, phydroxy benzoic acid, Gallic acid, and luteolin [19].

Permission: There is no permission for collecting the Aizoon plant from desert; Dr. Ashraf Nofal associate prof of Fungi and Plant Pathology, Environmental Studies and Research Institute, 
University of Sadat City, Who is collected the plant from Sadat city desert, Egypt, for student and research.

\section{Chemical composition of $\mathrm{SS} 430$ and its pretreatment}

SS 430 (SS430) belongs to a ferritic group of steels. It composed of carbon, manganese, phosphorus, sulfur, silicon, iron, chromium, and nickel. (Table 1).

Table 1. Chemical composition of SS430

\begin{tabular}{lllllllll}
\hline Element & $\mathrm{C}$ & $\mathrm{Mn}$ & $\mathrm{P}$ & $\mathrm{Cr}$ & $\mathrm{Si}$ & $\mathrm{S}$ & $\mathrm{Ni}$ & $\mathrm{Fe}$ \\
\hline \%Wt. & 0.12 & 1.0 & 0.045 & 16 & 1.0 & 0.03 & 0.5 & rest \\
\hline
\end{tabular}

Directly before test SS430 samples were polished with different grades of sandpaper 1000, 1500 and 2000 to a metallic shine, degreased with ethanol 98\% (ADWIC, Nasr Pharma, Egypt) and cleaned with double distilled water. Finally, dried at room temperature.

\section{Mass reduction (MR) measurements}

MR tests were fulfilled based on the American society for testing and materials (ASTM) standard procedure. All SS430 samples used in MR experiments were mechanically cut into a tetragonal shape of $20 \mathrm{~mm} \times 20 \mathrm{~mm} \times 2 \mathrm{~mm}$ dimensions with effective surface area $8 \mathrm{~cm}^{2}$. The concentration range of Aizoon extract in test solutions were selected to be 0-300 ppm. The pretreated SS430 sheets were weighed and then submerged in $100 \mathrm{~mL}$ of $2 \mathrm{M} \mathrm{HCl}$ solution with different concentrations of Aizoon extract (0-300 ppm) at 298-318 K for $3 \mathrm{~h}$. After the indicated time the SS specimens were removed from the corrosive medium, washed with double distilled water, dried, and weighed.

\section{Electrochemical measurements}

The behavior of Aizoon extract as an anti-corrosion for SS430 in $2 \mathrm{M} \mathrm{HCl}$ was further examined by PDP and (EIS) tests. All electrochemical experiments were performed at $298 \mathrm{~K}$ using threeelectrode setup consists of a saturated calomel electrode (SCE) as reference, platinum foil as an auxiliary electrode and SS430 as a working electrode (WE) in $100 \mathrm{~mL}$ glass cell. The (WE) was prepared as follows. One side of SS430 sheet $(10 \mathrm{~mm}$ x $10 \mathrm{~mm} \times 2 \mathrm{~mm})$ was connected to a copper wire for electric connection. Subsequently, the copper wire which attached to SS430 sheet was inserted into a glass tube and then fixed by epoxy resin to make the area of SS430 exposed to the solutions is $1 \mathrm{~cm}^{2}$. Prior to measurements the samples were pretreated as mentioned before and the potential of the electrode was permitted to stabilize for $30 \mathrm{~min}$ in order to reach a steady state. For PDP test the potential was scanned from -0.5 to $0.5 \mathrm{~V}$ against open circuit potential (OCP) with scan rate $0.5 \mathrm{mVs}^{-1}$. AC impedance tests were carried out in a frequency range from $0.1 \mathrm{~Hz}$ to $10^{-}$

${ }^{5} \mathrm{~Hz}$ with an amplitude of $10 \mathrm{mV}$ peak to peak under open circuit condition. The experimental data 
collected from EIS measurements were analyzed based on the equivalent circuit. However, polarization resistances $R_{p}$, were estimated from the distance between the high-and low-frequency intercepts with the abscissas for the impedance spectra. All electrochemical tests were performed using Gamry instrument Potentiostat/ Galvanostat/ ZRA (PCI4-G750). The utilized software is DC105 for PDP, EIS300 for EIS and the data obtained were analyzed using Echem analyst v 5.5 software.

\section{Surface Characterization}

SS430 samples used for surface analysis were submerged in extract-free acid and acid containing $300 \mathrm{ppm}$ of Aizoon extract $298 \mathrm{~K}$ for $3 \mathrm{~h}$. After that, the samples were removed cleaned with double distilled water many times to remove any residue, and dried. The surface morphology of SS430 samples was characterized using scanning electron microscope (SEM) (JOEL 840, Japan), atomic force microscope (AFM). The adsorption of Aizoon extract on SS430 surface was investigated by Fourier transform infrared spectroscopy (FTIR) Thermo Fisher Nicolet IS10, USA in the spectral range of $400-4000 \mathrm{~cm}^{-1}$. The FTIR spectra were recorded for SS430 surface before and after immersion $3 \mathrm{~h}$ in acid-Aizoon mixture $(300 \mathrm{ppm}$ Aizoon $+2 \mathrm{M} \mathrm{HCl})$ and then compared to the spectra of Aizoon extract. Before FTIR experiment, the SS430 sample which immersed in acid-Aizoon mixture was rinsed several times with ethanol and water to remove any Aizoon residue from the SS430 surface.

\section{Result and Discussion}

\section{Mass reduction (MR) tests}

The MR values, corrosion rate (CR), inhibition percent (I \%), and surface coverage ( $\theta$ ) for SS430/ $2 \mathrm{M} \mathrm{HCl}$ system in the presence of different Aizoon concentrations (0-300 ppm) at (298-318 K) which obtained from MR experiments after $3 \mathrm{~h}$ immersion were summarized in Table 2 and calculated through the following equations.

$$
M L=m_{2}-m_{1}
$$

where $m_{1}$ and $m_{2}$ are the weights of SS430 sheet prior and after submersion in the test solution, respectively.

$$
C R=87.6 \times \frac{M R}{D A t}
$$

where MR is the loss in mass, $\mathrm{D}$ is the alloy density, $\mathrm{t}$ is the submersion time and $\mathrm{A}$ is the active area exposed to the test solution.

$$
\begin{aligned}
& \theta=\frac{C R_{1}-C R_{2}}{C R_{1}} \\
& I \%=\frac{C R_{1}-C R_{2}}{C R_{1}} \times 100
\end{aligned}
$$

where $\mathrm{CR}_{1}$ and $\mathrm{CR}_{2}$ are the corrosion rates of SS430 without and with Aizoon extract, respectively. Figure 1(a) shows the mass reduction-time curves for SS430/2M HCl in different Aizoon concentrations at $298 \mathrm{~K}$ similar curves were done for other temperatures (not shown). It was observed that the addition of Aizoon extract was accompanied by decreases in the mass reduction and corrosion rate, on the other hand, the surface coverage and the inhibition percent increased 
where it reached to $95.8 \%$ in 300 ppm Aizoon concentration. This confirms the inhibition characteristic of Aizoon extract for protecting SS430 corrosion in acidic medium. Raising temperature of the test solutions leads to a significant increase in mass reduction and corrosion rate of SS430 in all Aizoon concentrations compared to those at $298 \mathrm{~K}$ (see Table 2). Therefore, the $\%$ I of the extract decreased with raising the temperature as it appears in Fig. 1(b) which summarize the inhibition efficiency as a function of temperature for all Aizoon concentrations. This can be explained by the decrease in the adsorption of Aizoon compounds on SS340 surface at high temperatures. However, the Aizoon extract shows an inhibition percent at $318 \mathrm{~K}$ reached to $85.6 \%$ in 300 ppm extract.
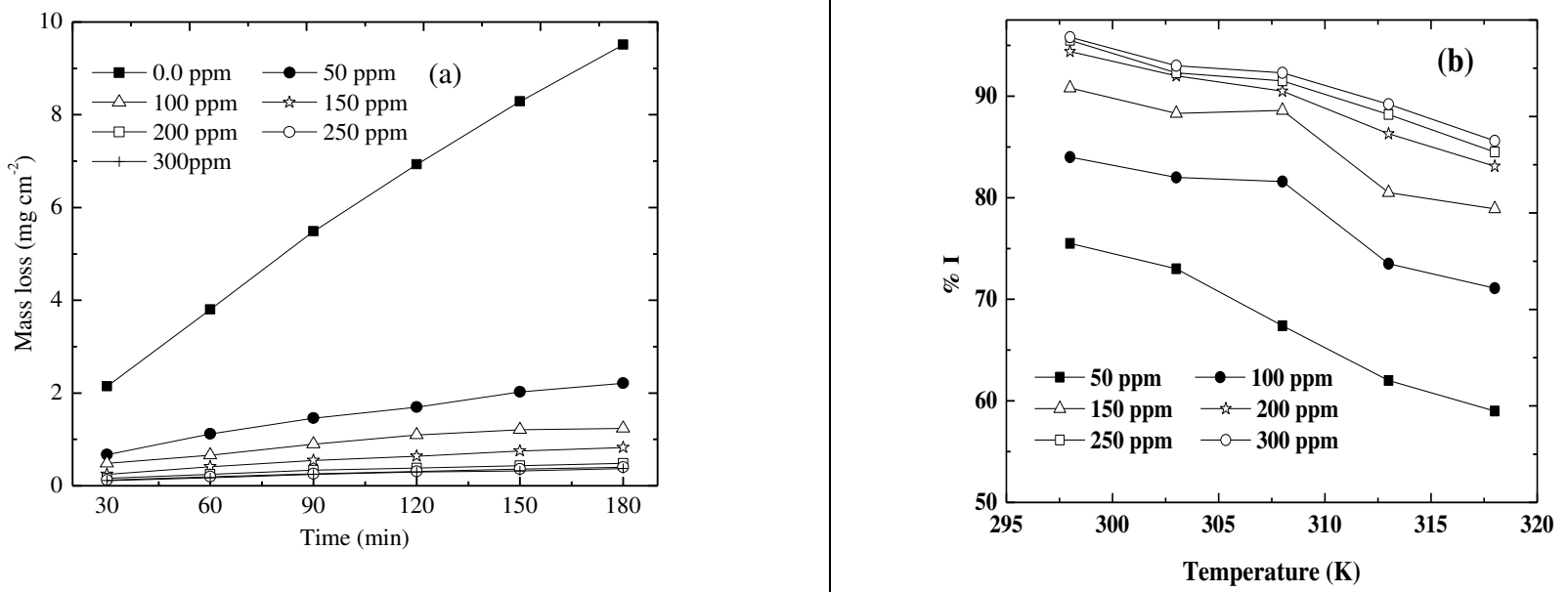

Figure 1. (a) MR-time curves at $298 \mathrm{~K}$ and (b) inhibition percent as a function of temperature for $\mathrm{SS430/2M} \mathrm{HCl} \mathrm{in} \mathrm{the} \mathrm{presence} \mathrm{of} \mathrm{various} \mathrm{Aizoon} \mathrm{concentrations.}$

Table 2. Dissolution parameters at different temperatures for SS430/2M HCl system in different Aizoon concentrations measured from mass reduction test.

\begin{tabular}{|c|c|c|c|c|c|}
\hline $\begin{array}{c}\text { Temp. } \\
\mathrm{K}\end{array}$ & $\begin{array}{c}\text { Conc., } \\
(\mathrm{ppm})\end{array}$ & $\begin{array}{c}\text { Mass red., } \\
\mathrm{mg}\end{array}$ & $\begin{array}{c}\mathrm{CR}, \\
\mathrm{mmy}^{-1}\end{array}$ & $\theta$ & $\% \mathrm{I}$ \\
\hline \multirow{4}{*}{298} & 0.0 & 52.7 & 39.2 & --- & ---- \\
\cline { 2 - 6 } & 50 & 12.9 & 9.6 & 0.755 & 75.5 \\
\cline { 2 - 6 } & 100 & 8.8 & 6.2 & 0.84 & 84 \\
\cline { 2 - 6 } & 150 & 5.1 & 3.6 & 0.908 & 90.8 \\
\cline { 2 - 6 } & 200 & 3.1 & 2.2 & 0.944 & 94.4 \\
\cline { 2 - 6 } & 250 & 2.5 & 1.8 & 0.955 & 95.5 \\
\hline
\end{tabular}




\begin{tabular}{|c|c|c|c|c|c|}
\hline & 300 & 2.4 & 1.7 & 0.958 & 95.8 \\
\hline \multirow{7}{*}{303} & 0.0 & 97.3 & 72.4 & --- & --- \\
\hline & 50 & 32.2 & 22.7 & 0.73 & 73 \\
\hline & 100 & 21.2 & 14.97 & 0.82 & 82 \\
\hline & 150 & 13.8 & 9.7 & 0.883 & 88.3 \\
\hline & 200 & 9.5 & 6.7 & 0.92 & 92 \\
\hline & 250 & 9.3 & 6.6 & 0.923 & 92.3 \\
\hline & 300 & 8.3 & 5.9 & 0.93 & 93 \\
\hline \multirow{7}{*}{308} & 0.0 & 173.1 & 128.7 & --- & --- \\
\hline & 50 & 59.4 & 41.9 & 0.674 & 67.4 \\
\hline & 100 & 33.4 & 23.6 & 0.816 & 81.6 \\
\hline & 150 & 20.7 & 14.6 & 0.886 & 88.6 \\
\hline & 200 & 17.2 & 12.1 & 0.905 & 90.5 \\
\hline & 250 & 15.4 & 10.9 & 0.915 & 91.5 \\
\hline & 300 & 13.9 & 9.8 & 0.923 & 92.3 \\
\hline \multirow{7}{*}{313} & 0.0 & 235 & 174.8 & --- & --- \\
\hline & 50 & 81.9 & 57.9 & 0.62 & 62 \\
\hline & 100 & 57.1 & 40.3 & 0.735 & 73.5 \\
\hline & 150 & 41.9 & 29.6 & 0.805 & 80.5 \\
\hline & 200 & 29.5 & 20.8 & 0.863 & 86.3 \\
\hline & 250 & 25.4 & 17.9 & 0.882 & 88.2 \\
\hline & 300 & 23.3 & 16.5 & 0.892 & 89.2 \\
\hline \multirow{7}{*}{318} & 0.0 & 287.7 & 213.9 & --- & --- \\
\hline & 50 & 146.8 & 103.7 & 0.59 & 59 \\
\hline & 100 & 105.2 & 74.3 & 0.711 & 71.1 \\
\hline & 150 & 84.1 & 59.4 & 0.789 & 78.9 \\
\hline & 200 & 61.6 & 43.5 & 0.831 & 83.1 \\
\hline & 250 & 56.4 & 39.8 & 0.845 & 84.5 \\
\hline & 300 & 52.6 & 37.2 & 0.856 & 85.6 \\
\hline
\end{tabular}

\section{Adsorption isotherms}

The main goal of identifying the type of adsorption isotherm for extract over a metal surface is to estimate the inhibition mechanism. Different isotherm models were tested for SS430 in HClAizoon mixture. It was found that the adsorption of Aizoon compounds on the surface of SS430 agree with Langmuir type obeying the following equation

$$
\frac{\theta}{1-\theta}=K_{a d s} \times C
$$

where $\mathrm{C}$ is the Aizoon concentration, and $\mathrm{K}_{\mathrm{ads}}$ is the equilibrium constant of the adsorption process. The relation between the $\frac{\theta}{1-\theta}$ and $\mathrm{C}$ in Fig. 2(a) gives a straight-line confirming Langmuir model for Aizoon adsorption at all studied temperatures. Therefore, a monolayer of Aizoon compounds is adsorbed on SS430 surface and there is no interaction between the adsorbed molecules on the alloy surface. The standard Gibbs function of adsorption-desorption process $\left(\Delta \mathrm{G}^{\mathrm{o}}\right.$ ads $)$ at different temperatures can be calculated according to the following equation: 


$$
K_{a d s}=55.5 e^{-\frac{\Delta G_{a d s}^{o}}{R T}}
$$

where 55.5 is the strength of water in the solution, $\mathrm{R}$ is $8.314 \mathrm{JK}^{-1} \mathrm{~mol}^{-1}$, $\mathrm{T}$ is the temperature in kelvin. It was found that the value of $\Delta \mathrm{G}^{\circ}$ ads is $-28.2 \mathrm{~kJ} \mathrm{~mol}^{-1}$ as calculated from Eq. (6) at $298 \mathrm{~K}$. The negative sign and numerical value for $\Delta \mathrm{G}^{\circ}$ ads confirm that the adsorption of Aizoon extract on SS430 surface in $2 \mathrm{M}$ hydrochloric acid is a spontaneous process belongs to physisorption and chemisorption mechanism (mixed adsorption) but mainly physisorption [20]. Moreover, the decrease in the equilibrium constant with temperature indicating that the rate of desorption increased at high temperature which is additional evidence that the adsorption of Aizoon compounds on SS430 surface is a physical in type (see Fig. 2(a)).

The enthalpy of adsorption of Aizoon compounds on SS430 surface in $2 \mathrm{M} \mathrm{HCl}\left(\Delta \mathrm{H}^{\circ}\right.$ ads $)$ at $298 \mathrm{~K}$ was calculated from Eq. (7)

$$
\log \frac{\theta}{1-\theta}=-\frac{\Delta H_{a d s}^{o}}{2.303 R T}+\text { const }
$$

Figure 2(b) shows the relation between $\log \frac{\theta}{1-\theta}$ and invers of absolute temperatures for SS430/2M $\mathrm{HCl}$ system with $300 \mathrm{ppm}$ Aizoon. The average value of $\Delta \mathrm{H}^{\circ}$ ads was evaluated from the slope of the fitted line and was equal $-49.9 \mathrm{~kJ} \mathrm{~mol}^{-1}$. Therefore, extract adsorption on the SS430 surface accompanied by releasing heat, confirming the spontaneity of the adsorption process. The entropy at $\operatorname{STP}\left(\Delta \mathrm{S}^{\circ}\right.$ ads $)$ for adsorption process can be estimated from the following equation:

$$
\Delta S_{a d s}^{o}=\frac{\Delta H_{a d s}^{o}-\Delta G_{a d s}^{o}}{T}
$$

The value of $\Delta \mathrm{S}^{\circ}$ ads is $-72.8 \mathrm{~J} \mathrm{~mol}^{-1} \mathrm{~K}^{-1}$, demonstrating increase in the order of the system as a result of adsorption of Aizoon compounds on SS430 surface and the adsorption process is favorable at low temperature.

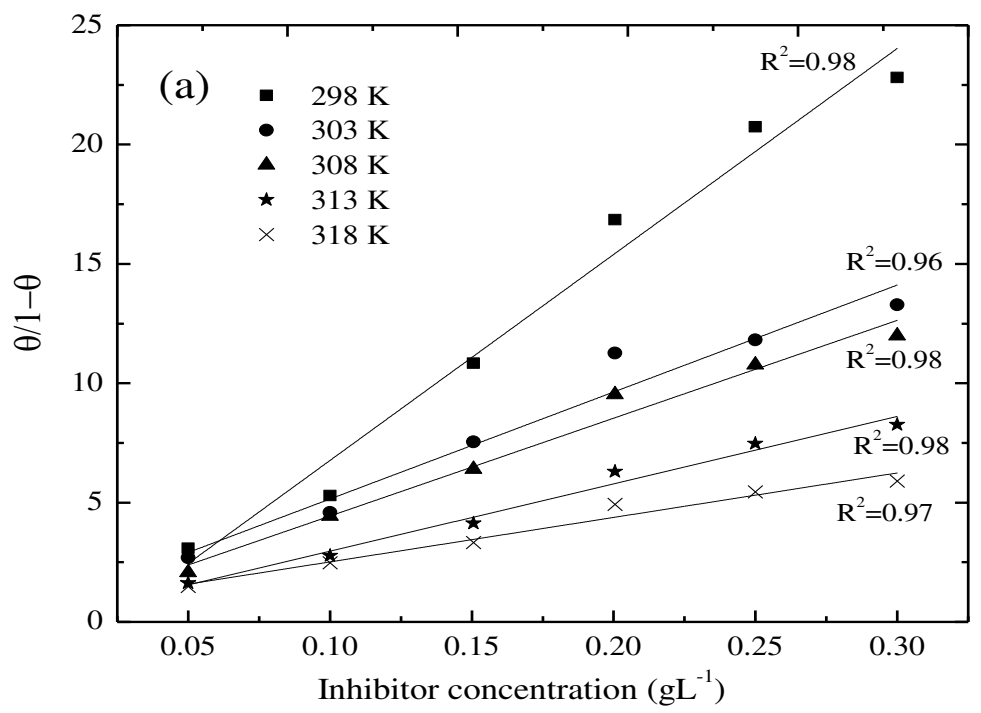




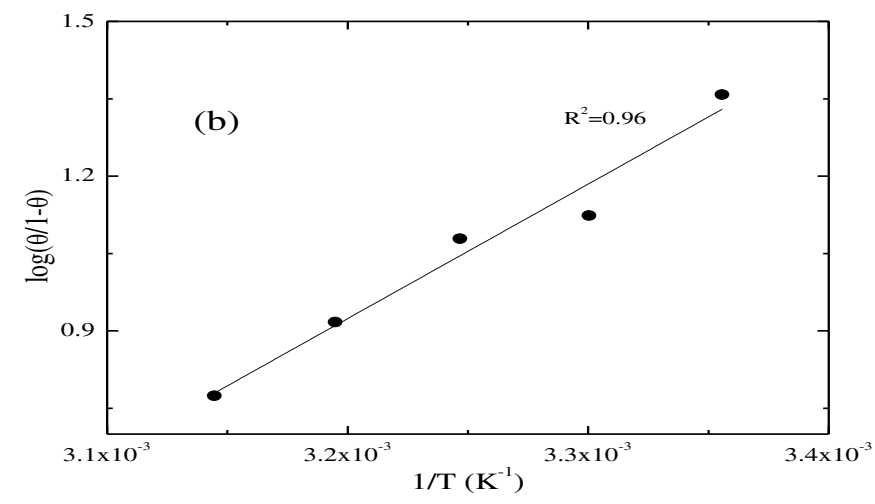

Figure 2. (a) Langmuir relation for adsorption of Aizoon on the surface of SS430 in $2 \mathrm{M} \mathrm{HCl}$ at different temperatures. (b) The relation between $\log (\theta / 1-\theta)$ and $(1 / \mathrm{T})$ for SS430/2M HCl system with 300 ppm Aizoon.

\section{Kinetic and thermodynamic parameters}

Arrhenius equation can be used to calculate the activation energy for corrosion process demonstrating the relation between $C R$ and activation energy $\left(E^{*}{ }_{a}\right)$ as in Eq. (9)

$$
C R=A e^{-\frac{E_{a}^{*}}{R T}}
$$

where $\mathrm{CR}$ in $\mathrm{mm} / \mathrm{y}$ obtained from mass reduction test, $\mathrm{A}$ is the pre-exponential constant. The logarithm of CR was plotted as a function of 1/T (see Fig. 3(a)). The slope of the fitted lines gives the $\left(\mathrm{E}^{*}{ }_{\mathrm{a}}\right)$ for corrosion process of SS430/2M HCl system at different Aizoon concentrations. The increase in $\left(\mathrm{E}_{\mathrm{a}}^{*}\right)$ for corrosion process with increasing Aizoon content in the corrosive medium, as shown in the Table 3, means that the Aizoon extract hindrance the corrosion of SS430 because of adsorption of Aizoon compounds on the SS430 surface.

The thermodynamic parameter of activation $\Delta \mathrm{H}^{*}$ and $\Delta \mathrm{S}^{*}$ for the corrosion process were calculated from Eq. (10).

$$
C R=\frac{R T}{N h} e^{\frac{\Delta S^{*}}{R}} \times e^{-\frac{\Delta H^{*}}{R T}}
$$

where $\mathrm{h}$ is Planck's constant and $\mathrm{N}$ is Avogadro's number. Figure 3(b) is the relation between $\log$ $(\mathrm{CR} / \mathrm{T})$ and $(1 / \mathrm{T}) . \Delta \mathrm{H}^{*}$ and $\Delta \mathrm{S}^{*}$ for SS430 corrosion in $2 \mathrm{M}$ hydrochloric acid with different Aizoon concentrations were estimated from the slope and the intercept of the fitted line in Fig. 3(b), respectively and their values are summarized in Table 3 . The positive values of $\Delta H^{*}$ both in the absence and presence of extract reflect the endothermic nature of SS430 dissolution process and indicates that the dissolution of SS430 is difficult [21]. However, an increment was observed in the positive value of $\Delta \mathrm{H}^{*}$ with increasing the extract content in the corrosive medium indicating that the corrosion reaction needs higher energy to proceed which confirm the inhibition effect of Aizoon extract. It was observed in Table 3 that $\mathrm{E}_{\mathrm{a}}^{*} \Delta \mathrm{H}^{*}$ vary in the same manner but the values of $\Delta \mathrm{H}^{*}$ are lower than that of $\mathrm{E}_{\mathrm{a}}^{*}$. This reported before that the corrosion process must involve a 
gaseous reaction [22]. Furthermore, the value of $\Delta \mathrm{S}^{*}$ is positive and increases significantly in the corrosive medium containing the Aizoon extract compared to that in free acid solution (see Table 3 ). This elucidates that the disorder increment occurs in transferring from reactants to the activated complex in the metal/solution interface. This is due to the replacement of solvent molecules in the medium which increase the randomness of the system.

Table 3. Activation factors for SS430 corrosion without and with various concentrations of Aizoon extract in $2 \mathrm{M} \mathrm{HCl}$.

\begin{tabular}{|c|c|c|c|}
\hline $\begin{array}{c}\text { Concentration } \\
(\mathrm{ppm})\end{array}$ & $\begin{array}{c}\mathrm{E}_{\mathrm{a}}^{*} \\
\left(\mathrm{~kJ} \mathrm{~mol}^{-1}\right)\end{array}$ & $\begin{array}{c}\Delta \mathrm{H}^{*} \\
\left(\mathrm{~kJ} \mathrm{~mol}^{-1}\right)\end{array}$ & $\begin{array}{c}\Delta \mathrm{S}^{*} \\
\left(\mathrm{~J} \mathrm{~mol}^{-1} \mathrm{~K}^{-1}\right)\end{array}$ \\
\hline Blank & 67.6 & 65.1 & 65.0 \\
\hline 50 & 90.0 & 87.4 & 68.4 \\
\hline 100 & 94.0 & 91.4 & 77.9 \\
\hline 150 & 106.0 & 103.4 & 113.6 \\
\hline 200 & 112.1 & 109.6 & 130.6 \\
\hline 250 & 113.6 & 111.0 & 134.4 \\
\hline 300 & 113.7 & 111.1 & 134.0 \\
\hline
\end{tabular}

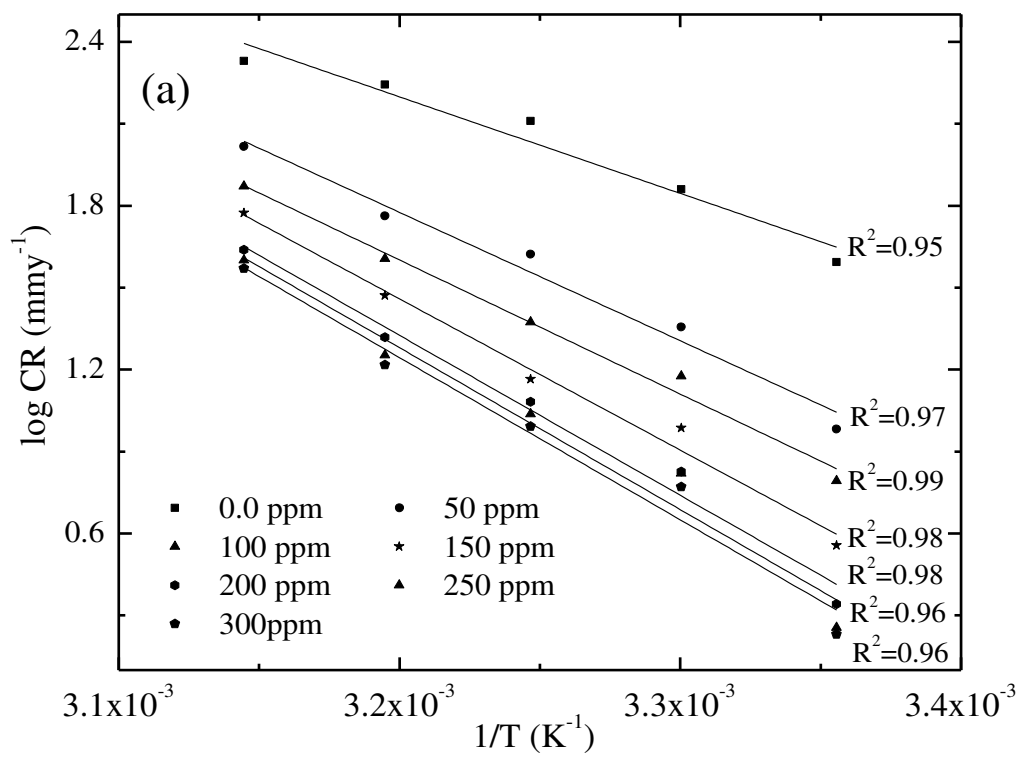




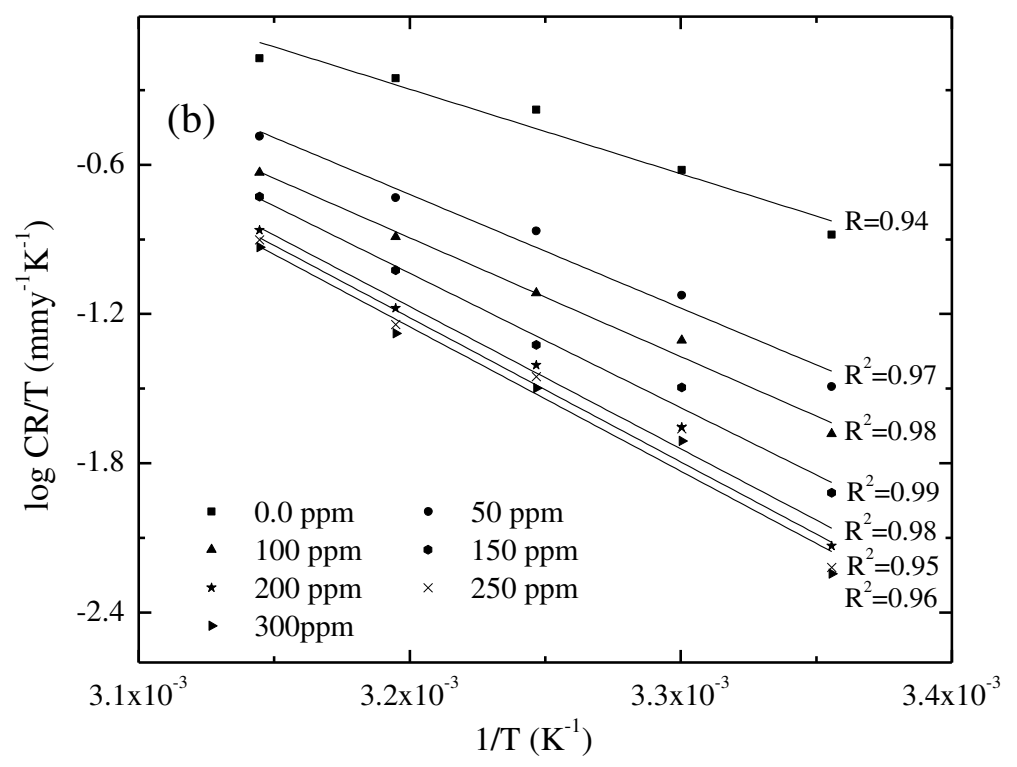

Figure 3. Arrhenius plots of (a) $\log \mathrm{CR}$ and (b) $\log \mathrm{CR} / \mathrm{T}$ against $1 / \mathrm{T}$ for $\mathrm{SS} 430 / 2 \mathrm{M} \mathrm{HCl}$ in different Aizoon concentrations.

\section{Electrochemical impedance spectroscopy (EIS) tests}

The corrosion action of SS430/2M HCl system in the presence of different Aizoon concentrations (0-300 ppm) was studied by EIS at $298 \mathrm{~K}$ and the spectra were depicted in Fig. 4 (a) and (b) for Nyquist and phase angle, respectively. The impedance spectra for SS430 in 2M HCl shows twotime constants, one of charge transfer reaction and the other is for a small conductive loop at the low-frequency region. On the other hand, the spectra for the $\mathrm{SS} 430 / \mathrm{HCl}$ system in the presence of the extract exhibit an additional time constant at the high-frequency region; which related to film formation and this capacitive loop become more noticeable at high extract concentration and overlapped with charge transfer loop at low concentration of extract (see Figure 4(a)). The inductive loop at low frequency domain could be related to the relaxation process of adsorbed species as hydrogen cation, chloride anion and/or Aizoon compounds on the electrode surface, or decay of the passive layer on SS430 surface at low frequency [23-26]. Different electrical circuits were examined to fit the data collected from EIS measurement. The circuits in the Figure 5 (a) and (b) showed the best agreement with the data obtained from EIS for SS430/HCl system in the blank and acid solution containing the extract, respectively. The equivalent circuit components are resistance of the solution $\left(R_{s}\right)$, film resistance $\left(R_{f}\right)$, film capacitance $\left(C_{f}\right)$, constant phase element $(\mathrm{CPE})$, charge transfer resistance $\left(\mathrm{R}_{\mathrm{ct}}\right)$ and resistance $\left(\mathrm{R}_{\mathrm{I}}\right)$ associated with the inductive element (L). The capacitor in the electrical circuit was replaced by (CFE) to compensate for surface heterogeneity. The capacitance double layer $\left(\mathrm{C}_{\mathrm{dl}}\right)$, and inhibition percent (I\%) were calculated using the following equations 


$$
C_{d l}=\left[Y_{0}\left(R_{c t}\right)^{1-n}\right]^{1 / n}
$$

where $\left(\mathrm{Y}_{0}\right)$ is the proportional factor, $\left(\mathrm{R}_{\mathrm{ct}}\right)$ charge transfer resistance and $(\mathrm{n})$ is an exponential term which is surface morphology dependent.

$$
I \%=100 \times\left(\frac{R_{p}-R^{\circ} p}{R_{p}}\right)
$$

where $\mathrm{R}_{\mathrm{p}}$ and $\mathrm{R}_{\mathrm{p}}^{\mathrm{o}}$ are the polarization resistance with and without Aizoon extract, respectively.

$$
\begin{aligned}
& R_{p}=R_{c t}+R_{f} \\
& R_{p}^{\circ}=R_{c t}^{\circ}
\end{aligned}
$$

where $R_{c t}$ and $R^{o}{ }_{c t}$ are the charge transfer resistance with and without Aizoon extract, respectively and $R_{f}$ is the film resistance. Extracted parameters were represented in Table 4. It was observed that the polarization resistance (Rp) for SS430/2M HCl in different Aizoon concentration that measured from EIS was in a great consistency with that estimated from potentiodynamic measurements in the next section (see Table 4 and 5). Both the film and charge transfer resistance increased with increasing Aizoon concentration, see Table 4, demonstrating the decrease in the rate of SS430 corrosion in the existence of Aizoon extract. However, the $\mathrm{R}_{\mathrm{ct}}$ is much higher than $\mathrm{R}_{\mathrm{f}}$ in all Aizoon concentrations, indicating that the corrosion mechanism still under control of charge transfer and only a thin film was formed [27]. The value of the exponential term (n) which reflects the surface homogeneity is decreased significantly in the existence of Aizoon compounds compared to its value in the free acid confirming the film formation on SS430 surface. In other words, the surface of SS430 becomes less homogenous because of the growth of a thin film from extract on it. Moreover, the value of (n) shows a slight increase with increasing Aizoon concentration this means that the film formation increased which leads to enhancement in the surface homogeneity. The capacitance double layer value decreases with increase of Aizoon extract concentration because of increase in the thickness of the electrical double layer formed on the SS430 surface and/or decrease in dielectric constant of corrosive medium which can be explained by removal of water molecules by extract compounds according to the Helmholtz equation $[28,29]$.

$$
C_{d l}=\frac{\varepsilon \varepsilon^{\circ} A}{d}
$$

where $\varepsilon$ is the corrosive medium dielectric constant, $\varepsilon^{0}$ is the permittivity of the vacuum, $d$ is the protective layer thickness and $\mathrm{A}$ is the electrode surface area. Furthermore, the maximum phase angle at high-frequency domain moved to more negative value with rising the extract amount in the corrosive medium (see Fig. 4(b)), this means that Aizoon act as a barrier providing a better protection for SS430 from corrosion in high extract concentration. 


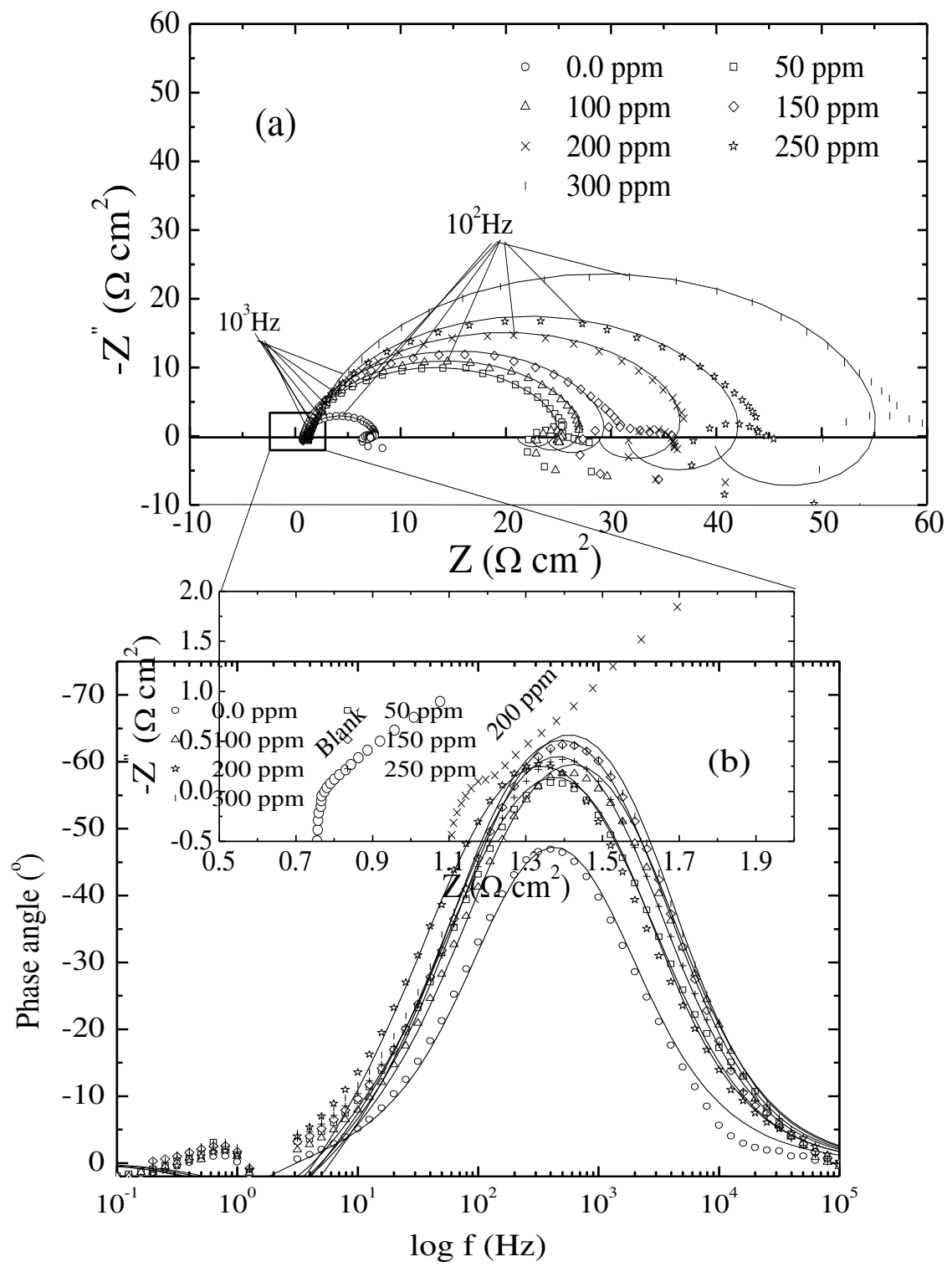

Figure 4(a). Impedance spectra and (b) Phase angle vs. $\log f$ of the impedance spectra for SS430 in $2 \mathrm{M} \mathrm{HCl}$ with different Aizoon concentrations (0-300 ppm) at $298 \mathrm{~K}$. The symbols represent the experimental data, while solid lines are the fitted curves using the modified equivalent circuit. 

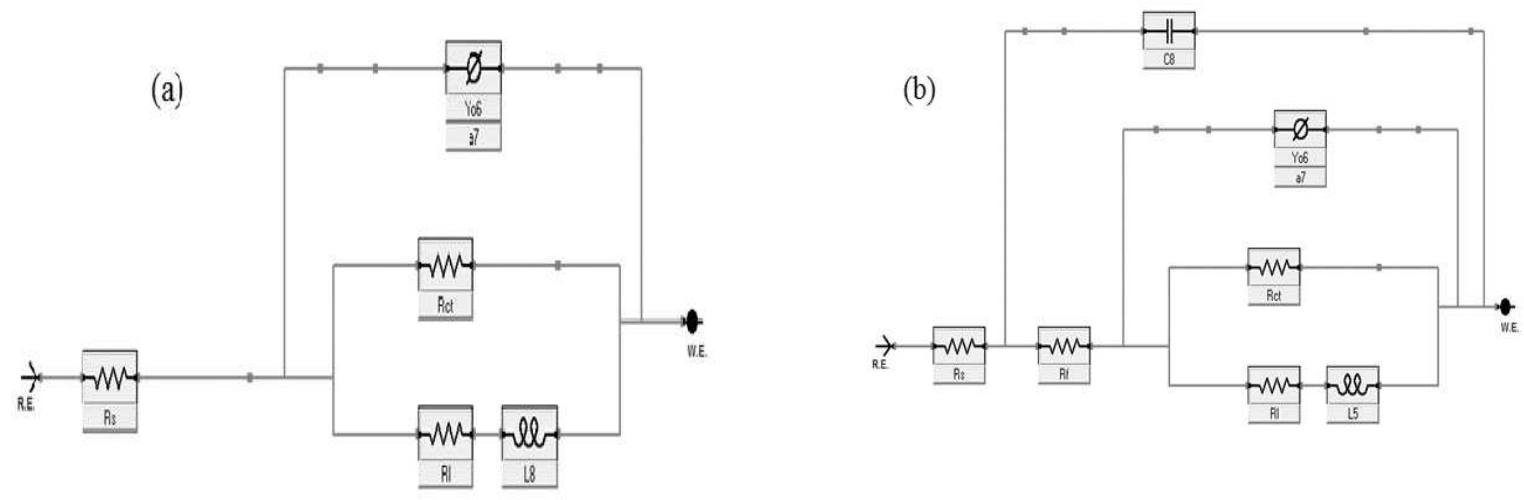

Figure 5. Equivalent circuit diagrams used to fit impedance data for SS430/2M HCl in (a) without and (b) with extract at $298 \mathrm{~K}$.

Table 4. Electrochemical parameters obtained from EIS test for the corrosion of SS430 in $2 \mathrm{M} \mathrm{HCl}$ with various concentrations of Aizoon at $298 \mathrm{~K}$

\begin{tabular}{|c|c|c|c|c|c|c|c|c|c|c|c|}
\hline $\begin{array}{c}\text { Conc. } \\
\text { ppm }\end{array}$ & $\begin{array}{c}\mathrm{R}_{\mathrm{s}} \\
\mathrm{ohm} \\
\mathrm{cm}^{2}\end{array}$ & $\begin{array}{c}R_{\mathrm{f}} \\
\mathrm{ohm} \\
\mathrm{cm}^{2} \\
\end{array}$ & $\begin{array}{c}\mathrm{R}_{\mathrm{ct}} \\
\mathrm{ohm} \\
\mathrm{cm}^{2}\end{array}$ & $\begin{array}{c}R_{\mathrm{L}} \\
\mathrm{ohm} \\
\mathrm{cm}^{2} \\
\end{array}$ & $\begin{array}{c}\mathrm{R}_{\mathrm{p}}= \\
\mathrm{R}_{\mathrm{ct}}+\mathrm{R}_{\mathrm{f}} \\
\mathrm{ohm} \mathrm{cm} \mathrm{cm}^{2}\end{array}$ & $\mathrm{~L}(\mathrm{H})$ & $\begin{array}{c}\mathrm{C}_{\mathrm{f}} \times 10^{-6} \\
(\mathrm{~F})\end{array}$ & $Y_{o} \times 10^{-6}$ & $\mathrm{n}$ & $\begin{array}{l}\mathrm{C}_{\mathrm{dl}} \\
(\mathrm{F})\end{array}$ & I $\%$ \\
\hline 0.0 & 0.788 & - & 6.950 & 17.9 & 6.950 & 9.408 & - & 384 & 0.896 & 192.0 & --- \\
\hline 50 & 1.031 & 0.630 & 25.0 & 88.0 & 25.630 & 6.980 & 49.8 & 328 & 0.710 & 46.2 & 72.9 \\
\hline 100 & 0.966 & 0.970 & 26.7 & 88.2 & 27.67 & 6.971 & 35.8 & 223 & 0.726 & 32.0 & 74.9 \\
\hline 150 & 0.810 & 1.351 & 28.84 & 88.4 & 30.191 & 7.637 & 45.6 & 205 & 0.712 & 25.7 & 77.0 \\
\hline 200 & 1.178 & 1.877 & 36.2 & 85.9 & 38.077 & 7.250 & 20.5 & 155 & 0.734 & 23.7 & 81.8 \\
\hline 250 & 1.400 & 2.191 & 41.5 & 88.7 & 43.691 & 9.910 & 20.2 & 125 & 0.738 & 19.3 & 84.1 \\
\hline 300 & 1.700 & 3.591 & 52.83 & 100 & 56.421 & 10.40 & 25.6 & 95 & 0.753 & 16.7 & 87.7 \\
\hline
\end{tabular}

Potentiodynamic polarization (PDP) tests

PDP measurements were done for SS430 electrode in $2 \mathrm{M} \mathrm{HCl}$ to evaluate the electrochemical kinetic behavior with different concentrations from Aizoon extract. Figure 6 shows the Tafel curves for SS430/HCl system in different concentrations of Aizoon (0-300 ppm). The corrosion potential $\left(\mathrm{E}_{\text {corr }}\right)$ and corrosion current density $\left(\mathrm{i}_{\mathrm{corr}}\right)$ were estimated from the coordinates of the point when the linear part of the anodic and cathodic curve intersected. All the electrokinetic parameters obtained from Tafel extrapolation were summarized in Table 5. It was noticed that the cathodic curves in the Tafel plot for SS430 are parallel in both the extract-free acid and in different 
concentrations of extract which shows that the addition of Aizoon extract does not alter the mechanism of hydrogen reduction reaction. On the other hand, the anodic Tafel slope decreased significantly in extract-acid mixture compared to that in blank corrosive medium, confirming the change in metal dissolution mechanism by addition of the extract (see Fig.6 and Table 5). However, the addition of extract leads to a considerable shift in the $\mathrm{i}_{\text {corr }}$ to lower value compared with that in free acid and it is concentration dependent. Moreover, the value of $\mathrm{E}_{\text {corr }}$ for SS430/HCl system was changed to lower negative values with Aizoon extract this means that the SS430 in extract-free acid starts to corrode first. In other words, the presence of the extract retards the SS430 corrosion in $2 \mathrm{M}$ hydrochloric acid solution (see Table 5). The inhibition percent (I\%) and ( $\theta)$ in different extract concentrations were calculated using the following equations.

$$
\begin{aligned}
& \theta=1-\frac{i_{\text {corr }}}{i_{\text {corr }}^{o}} \\
& I \%=\theta \times 100
\end{aligned}
$$

where, $\mathrm{i}^{\mathrm{o}}$ corr and $\mathrm{i}_{\text {corr }}$ were the corrosion current densities without and with Aizoon extract, respectively. It was found that the $\%$ I increased, and the CR decreased with increasing extract concentration which is comparable with that obtained from MR tests at $298 \mathrm{~K}$ (see Table 2 and 5). The values of $i^{o}$ corr (current at equilibrium) is comparable to the values of corrosion current densities ( $i_{\text {corr }}$ ) (Table 5). This illustrates that the constituent compounds of Aizoon extract were successfully adsorbed on the SS430 surface which in turn close the active sites and retard the rate of corrosion. Furthermore, the polarization resistances $\left(\mathrm{R}_{\mathrm{p}}\right)$ for $\mathrm{SS} 430 / \mathrm{HCl}$ system with different concentrations of Aizoon were calculated according to the following equation:

$$
R_{p}=\frac{\beta_{a} \beta_{c}}{2.303\left(\beta_{a}+\beta_{c}\right) i_{c o r r}}
$$

where, $i_{\text {corr }}$ is the corrosion current density. $\beta_{\mathrm{a}}$ and $\beta_{\mathrm{c}}$ are the anodic and cathodic Tafel slope, respectively. The $\mathrm{R}_{\mathrm{p}}$ increased significantly with increasing extract concentration, confirming the formation of the adsorbed layer from Aizoon extract on the SS430 surface which increased with increasing extract concentration, as it can be seen from the surface coverage $(\theta)$, hindering the rate of corrosion process for SS430 (see Table 5). The rate of SS430 dissolution and hydrogen devolution under different anodic and cathodic overpotential for SS430/2M HCl system in different extract concentrations were obtained from Faraday's equation and then depicted as a function of extract concentration at $298 \mathrm{~K}$ in Figure 7 (a) and (b), respectively. The rate of metal oxidation and hydrogen reduction were increased as expected with increasing the applied anodic or cathodic overpotential, respectively. The addition of extract leads to lower the rate of anodic and cathodic reaction at overpotential below $400 \mathrm{mV}$. This demonstrates that the Aizoon extract can be adsorbed on anodic and cathodic sites on SS430 surface. Otherwise, at anodic overpotential above $400 \mathrm{mV}$ there was no observable change in SS430 dissolution rate by addition of Aizoon extract (see Fig. 7 (a)). This means that the extract molecules were desorbed from anodic sites at that high anodic overpotential, resulting in no shift in the current density with the addition of different extract concentrations. However, above $400 \mathrm{mV}$ cathodic overpotential there was a considerable decrease in the hydrogen evolution rate with the addition of Aizoon extract to SS430/HCl system (see Fig. 7 (b)) which indicate that the components of Aizoon extract were 
strongly adsorbed to cathodic sites even at high overpotential. Moreover, the decrease in the rate of hydrogen evolution was higher with the addition of Aizoon extract compared to the decrease in SS430 dissolution rate as observed in Fig. 7 (a) and (b). Since the value of $E_{\text {corr }}$ with and without adding extract less than $85 \mathrm{mV}(46 \mathrm{mV})$, therefore, the extract acts as mixed type inhibitor tending to be superior for inhibiting the cathodic reaction $(\beta c>\beta a)$.

Table 5. Polarization parameter obtained from Tafel curves for SS430 in $2 \mathrm{M}$ HCl with different concentration of Aizoon at $298 \mathrm{~K}$

\begin{tabular}{|c|c|c|c|c|c|c|c|c|c|c|}
\hline $\begin{array}{c}\text { Conc., } \\
\text { ppm }\end{array}$ & $\begin{array}{c}-\mathrm{E}_{\text {corr }} \\
\text { mVvs. SCE }\end{array}$ & $\begin{array}{c}\beta_{a} \\
\mathrm{mV} / \mathrm{dec}\end{array}$ & $\begin{array}{c}-\beta_{c} \\
\mathrm{mV} / \mathrm{dec}\end{array}$ & $\begin{array}{c}i_{\text {corr }} \\
\mu \mathrm{A} / \mathrm{cm}^{2}\end{array}$ & $\begin{array}{c}\mathrm{CR} \\
\mathrm{mmy}^{-1}\end{array}$ & $\theta$ & $\% \mathrm{I}$ & $\begin{array}{c}R_{p} \\
\text { ohm cm }^{2}\end{array}$ & $(1-\alpha)$ & $\begin{array}{c}i_{o} \\
\mu \mathrm{A} / \mathrm{cm}^{2}\end{array}$ \\
\hline Blank & 528 & 91 & 126 & 3980 & 45.08 & - & - & 5.77 & 0.468 & 3370 \\
\hline 50 & 498 & 67 & 122 & 660 & 7.48 & 0.834 & 83.4 & 28.32 & 0.486 & 627 \\
\hline 100 & 501 & 58 & 118 & 621 & 7.03 & 0.843 & 84.4 & 27.24 & 0.503 & 570 \\
\hline 150 & 482 & 63 & 120 & 575 & 6.51 & 0.856 & 85.6 & 31.02 & 0.501 & 511 \\
\hline 200 & 487 & 66 & 120 & 511 & 5.79 & 0.872 & 87.2 & 36.04 & 0.494 & 460 \\
\hline 250 & 514 & 73 & 119 & 409 & 4.63 & 0.897 & 89.7 & 47.93 & 0.497 & 393 \\
\hline 300 & 510 & 71 & 121 & 280 & 3.17 & 0.93 & 93.0 & 69.55 & 0.490 & 246 \\
\hline
\end{tabular}

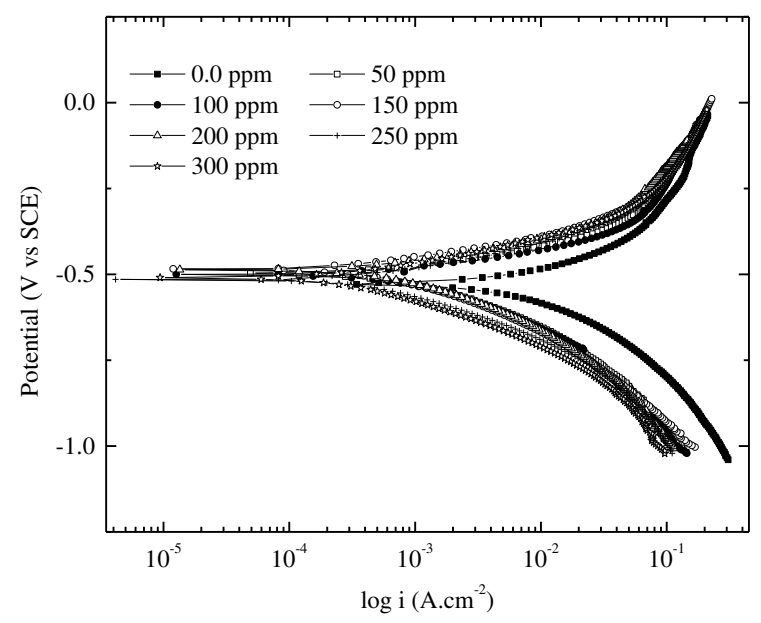

Figure 6. PDP curves for the dissolution of SS430 in $2 \mathrm{M} \mathrm{HCl}$ with different concentrations of Aizoon extract (0-300 ppm) at $298 \mathrm{~K}$

\section{Effect of extract on cathodic reaction}

The cathodic reaction for metal corrosion in an acidic medium is mainly a hydrogen evolution reaction (HER). The (HER) in acidic solution proceeds in three steps which are proton reduction (Volmer reaction), electrochemical desorption (Heyrovsky reaction) and/or chemical desorption (Tafel reaction) that is represented by the following equations. 
$H^{+}+e^{-} \rightleftharpoons H_{a d s}$

(Volmer reaction)

$H^{+}+H_{\text {ads }}+e^{-} \rightleftharpoons H_{2}$

(Heyrovsky reaction)

$H_{a d s}+H_{a d s} \rightleftharpoons H_{2}$

(Tafel reaction)

Thus, the whole reaction takes place through the integration of two probable reaction steps, Volmer-Heyrovsky and Volmer-Tafel. The Tafel slope is a good approximation for defining the rate-determining step for (HER). The kinetic models for (HER) proposed that the rate-determining step is Volmer, Heyrovsky or Tafel reaction if the Tafel slope is about 120,40 or $30 \mathrm{mV} \mathrm{dec}^{-1}$, respectively [28]. The estimated cathodic Tafel slopes in this study were in range 126.4-117.5 mV dec $^{-1}$ demonstrating that the rate-determining step for (HER) over SS430 electrode in $2 \mathrm{M} \mathrm{HCl}$ with or without Aizoon extract is Volmer step. The exchange current density $\left(i_{o}\right)$ and charge-transfer coefficient $(1-\propto)$ for (HER) for the examined systems were calculated from Tafel equation

$$
\begin{aligned}
\eta & =a+b \log i \\
b & =\frac{-2.303 R T}{(1-\alpha) n F} \\
a & =\frac{-2.303 R T}{(1-\alpha) n F} \times \log i_{o}
\end{aligned}
$$

where $\eta$ is the applied overpotential, $i$ is the current density, $b$ is the Tafel slope, $a$ is the intercept. $\mathrm{R}$ is $8.314 \mathrm{~J} \mathrm{~mol}^{-1} \mathrm{~K}^{-1}, \propto$ is the symmetry factor, $\mathrm{n}$ is the number of electrons exchanged and $\mathrm{F}$ is 96,485 Coulomb mol ${ }^{-1}$. As listed in the Table 5 the value of charge-transfer coefficients is all approaching to 0.5 which gives additional evidence that the Volmer step is the rate- determining step [30]. The exchange current density for (HER) over SS430 in $2 \mathrm{M} \mathrm{HCl}$ was decreased significantly by rising the concentration of Aizoon extract, indicating that the rate of (HER) decreases in the existence of Aizoon extract. Accordingly, as the Volmer step is the ratedetermining step for hydrogen evolution reaction, the active cathodic sites for hydrogen adsorption on SS430 electrode were decreased because of extract adsorption on these sites. In other word, the presence of extract decreases the surface area for hydrogen adsorption and slows down the charge transfer reaction.

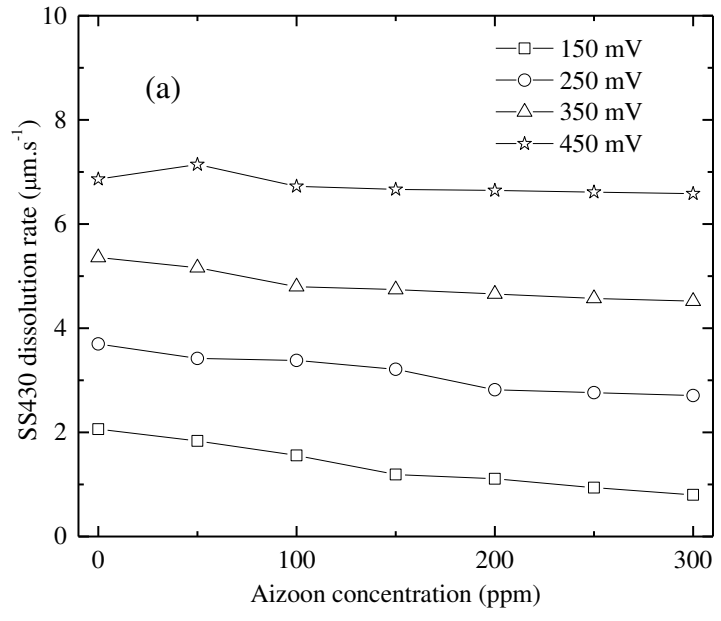




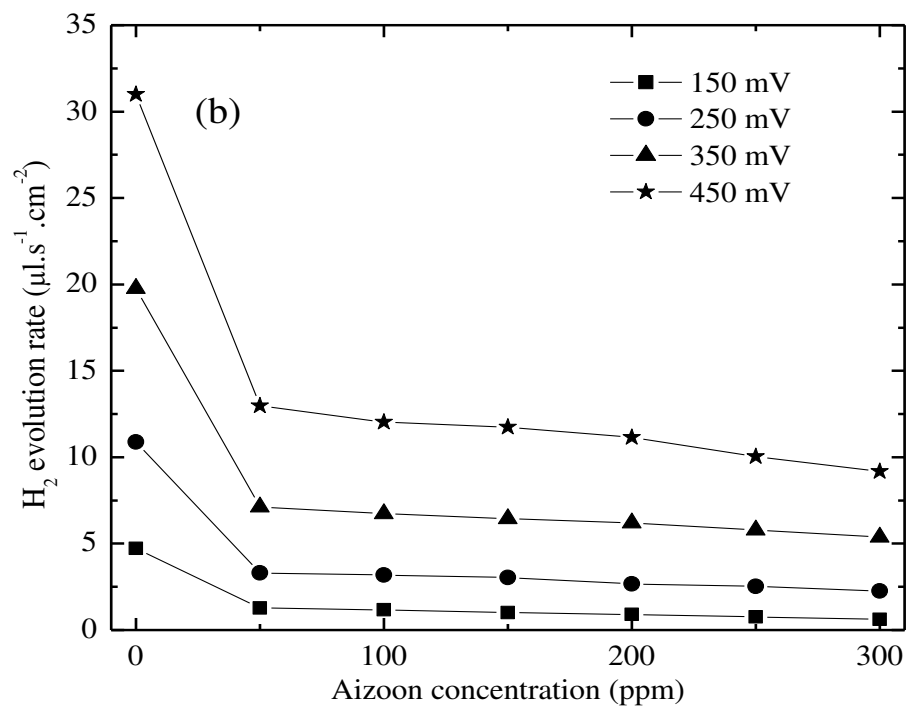

Figure 7 (a). Rate of SS430 dissolution and (b) hydrogen evolution under different anodic and cathodic overpotential, respectively for $\mathrm{SS340/HCl}$ system as a function of extract concentration at $298 \mathrm{~K}$

\section{Surface examination}

\section{Scanning electron microscope (SEM) analysis}

SEM test was conducted to elaborate a connection between the experimental data and the morphological shape of SS430 surface. Figure 8 (a), (b) and (c) are the SEM images for the aspolished SS430 surface and the surface after $3 \mathrm{~h}$ immersion in acid without extract and acid containing $300 \mathrm{ppm}$ of Aizoon extract at $298 \mathrm{~K}$, respectively. The image in Fig. 8(b) shows a noticeable crack on the alloy surface because of sever attack of the corrosive medium without extract. On the other hand, in the acid solution containing extract, the cracks disappeared and the surface of SS430 become smoother compared to the surface of SS430 in hydrochloric acid without extract (see Fig. 8(c)). This demonstrates that the Aizoon extract retarded the corrosion of SS430 through adsorption of its components on the SS430 surface.

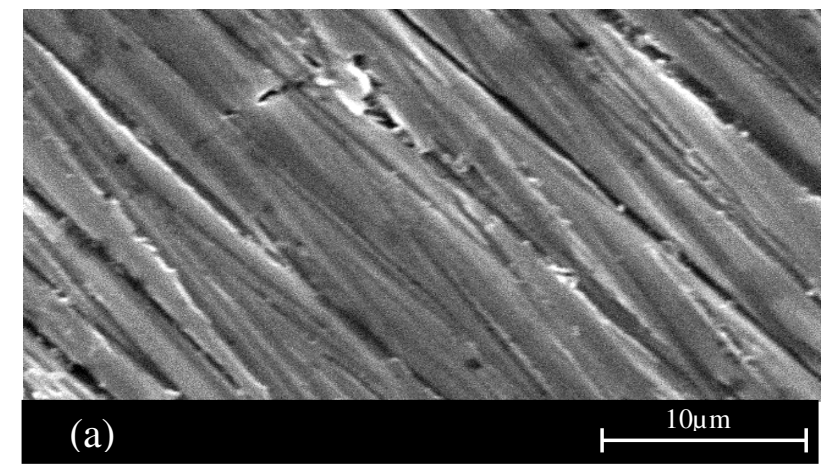



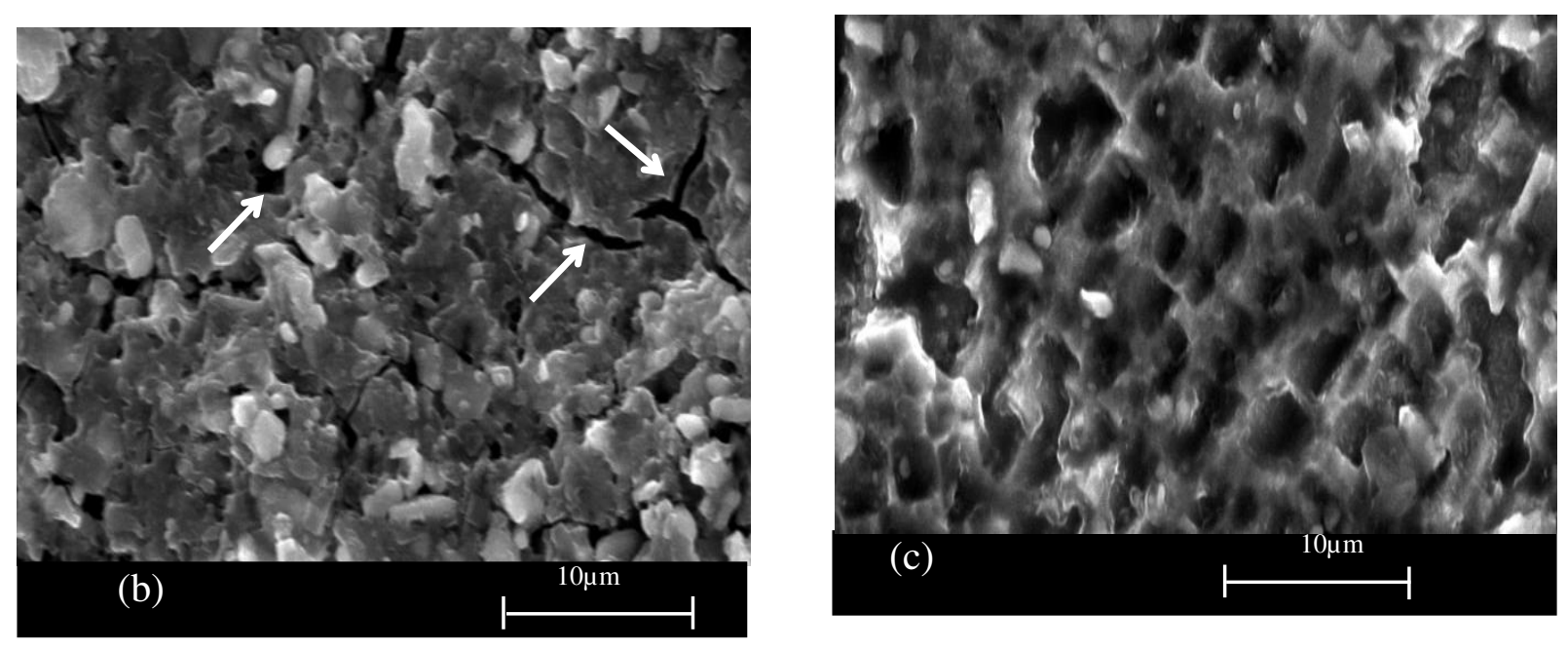

Figure 8. SEM micrographs of SS430 surface (a) before of immersion in $2 \mathrm{M} \mathrm{HCl}$ and after $15 \mathrm{~h}$ of immersion in (b) $2 \mathrm{M} \mathrm{HCl}$ and (c) $2 \mathrm{M} \mathrm{HCl}+300 \mathrm{ppm}$ of Aizoon extract at $298 \mathrm{~K}$

\section{Atomic force microscope (AFM) analysis}

The roughness of SS430 surface was examined by AFM. The three-dimensional AFM image for the pre-treated SS430 surface was shown in Fig. 9(a). It was found that the average surface roughness for polished SS430 is $18.52 \mathrm{~nm}$. After keeping SS430 for $3 \mathrm{~h}$ in corrosive medium without extract at $298 \mathrm{~K}$ the average surface roughness increased significantly to $174.18 \mathrm{~nm}$ while in the acid-Aizoon mixture (300 ppm Aizoon $+2 \mathrm{M} \mathrm{HCl}$ ) it decreased to $124.8 \mathrm{~nm}$ (see Fig 9(b) and (c)). The decrease in average surface roughness of SS430 when the extract was added to the corrosive medium is due to the adsorption of Aizoon on the SS430 surface retarding its corrosion.
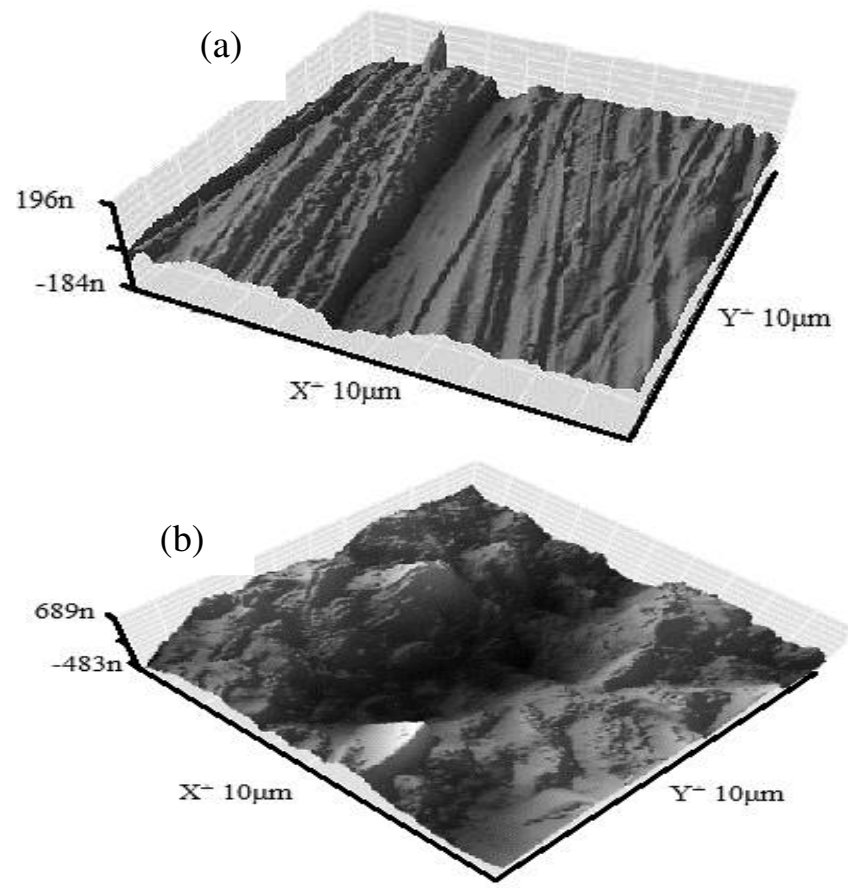


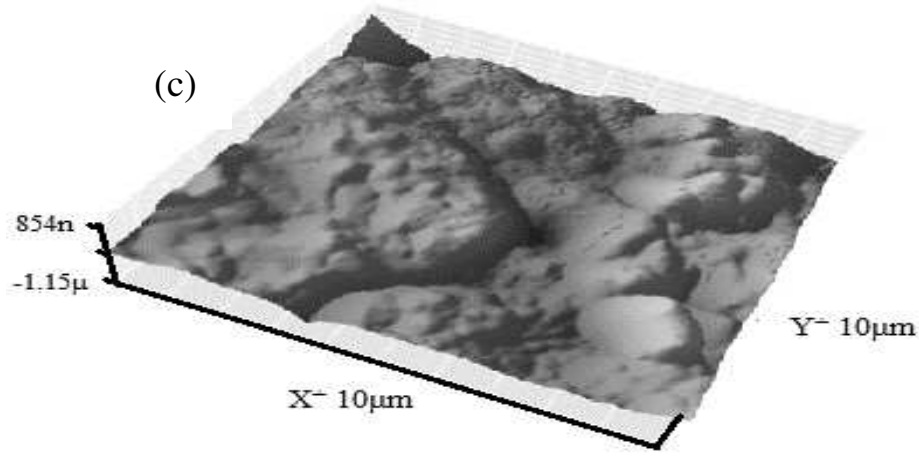

Figure 9. AFM 3D images for the surface of (a) the as-pretreated SS430 sample and after submersion in (b) $2 \mathrm{M} \mathrm{HCl}$ and (c) $2 \mathrm{M} \mathrm{HCl}+300 \mathrm{ppm}$ Aizoon for $15 \mathrm{~h}$ at $298 \mathrm{~K}$.

\section{Fourier transform infrared (FTIR) analysis}

FTIR measurements were conducted to obtain a clear evaluation for the possibility of the adsorption of a thin film from extract over SS430 surface. FTIR Spectra for the Aizoon extract and SS430 surface before and after submersion in mixture of $2 \mathrm{M} \mathrm{HCl}+300 \mathrm{ppm}$ extract for 3 hours at $298 \mathrm{~K}$ are presented in Fig. (10). It was observed that there are no peaks appeared when the alloy surface was examined before immersion in the mixture as in Fig. 10(a). However, the SS430 sample that was immersed in the mixture shows IR peaks like those for pure Aizoon extract with a noticeable change in both intensity and wave number (see Fig 10b and 10c). The broad band at $3355 \mathrm{~cm}^{-1}$, that related to $\mathrm{O}-\mathrm{H}$ stretching, was shifted to $3371 \mathrm{~cm}^{-1}$ after the submersion which means that the $\mathrm{O}-\mathrm{H}$ bond strength increased, and its bond length decreased that can be explained by the presence of adsorbed $\mathrm{O}$ atoms over SS430 surface [31]. Also, the peak related to C-H shifted from 2918 to $2921 \mathrm{~cm}^{-1}$. Moreover, the peak that belongs to the carbonyl group for pure Aizoon extract was in 1715 and shifted to $1730 \mathrm{~cm}^{-1}$ when examined over SS430 surface after immersion in HCl-Aizoon mixture accompanied with a decrease in its intensity which supports the possibility of adsorption of Aizoon compounds on SS430 surface [32,33]. In other words, the shift in peaks can be assigned to the interaction between extract molecules and active sites on metal surface. This means that the compounds of Aizoon were adsorbed successfully on SS430 surface confirming the formation of a protective thin film as discussed in EIS section. 


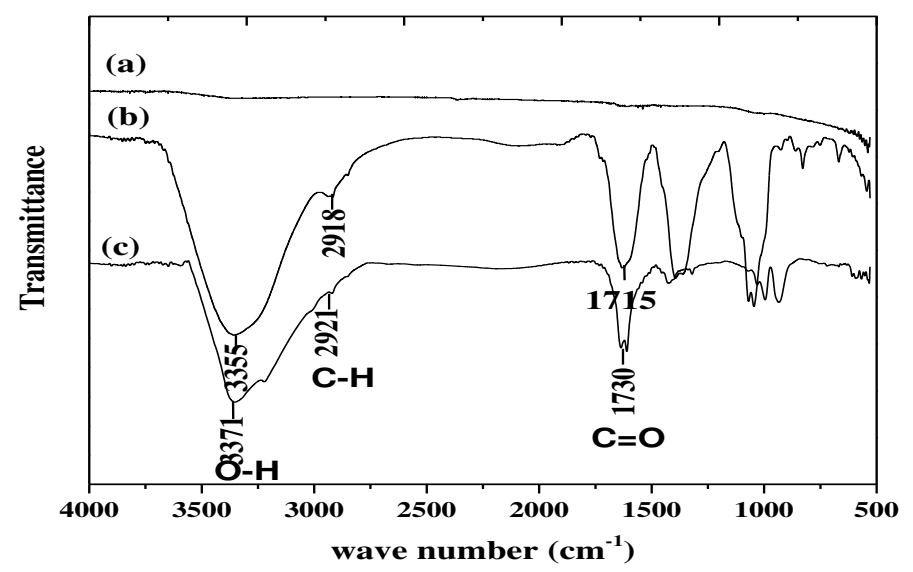

Figure 10. FTIR spectra for (a) as-pretreated SS430 surface, (b) Aizoon extract, and (c) SS430 surface after submersion in $2 \mathrm{M} \mathrm{HCl}+300$ ppm Aizoon for $3 \mathrm{~h}$ at $298 \mathrm{~K}$.

\section{Theoretical study results}

Luteolin considered the main components in Aizoon extract. So, we select it to carry out the Theoretical study on it

\section{Luteolin}<smiles>O=c1cc(-c2ccc(O)c(O)c2)oc2cc(O)cc(O)c12</smiles>

Table 6. The determined quantum chemical indicators

\begin{tabular}{|c|c|c|c|c|}
\hline Comp. & $\mathrm{E}_{\text {HOMO }}(\mathrm{ev})$ & $\mathrm{E}_{\text {LUMO }}(\mathrm{ev})$ & $\Delta \mathrm{E}(\mathrm{ev})$ & Dipole moment (Debye) \\
\hline Luteolin & -5.445 & -1.314 & 4.131 & 2.9624 \\
\hline
\end{tabular}

Comp. 


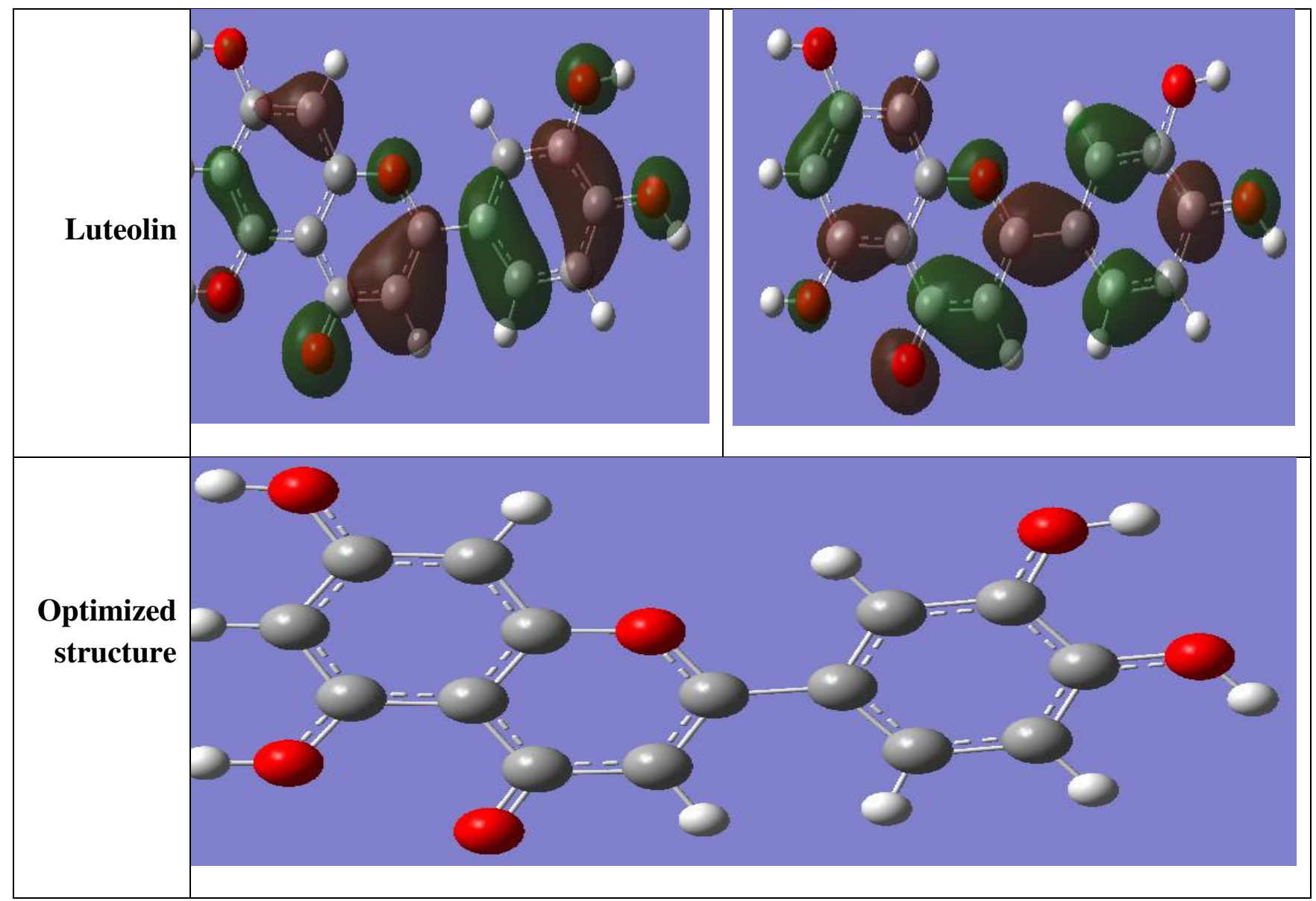

Figure 11. Frontier molecular orbital and electrostatic potential surface distribution for loaded form of Luteolin

Some quantum chemical parameters which are significant impact directly on electronic interaction between the metal surface and Luteolin inhibitor. Figure 11. Represents frontier molecular orbital and electrostatic potential surface distribution for loaded form of Luteolin. inhibitor. Higher $\mathrm{E}_{\mathrm{HOMO}}$ (highest occupied molecular orbital) of the adsorbent leads to higher electron donating ability. Thus, high values of $\mathrm{E}_{\mathrm{HO} о \mathrm{mo}}$ facilitate adsorption, and hence the inhibition efficiency is improved. The energy of the lowest unoccupied molecular orbital ( $\mathrm{E}_{\text {LUMO }}$ ) indicates the ability of the molecules to accept electrons. The lowering values of $\mathrm{E}_{\mathrm{LUMO}}$ lead to the more probable that the molecule would accept electrons. The dipole moment is a measure of the covalent bond polarity of the Luteolin compound. It is agreed that the large values of dipole moment enhance the adsorption propensity of the studied compound on metal surface. The determined quantum chemical indicators of Luteolin were shown in Table 6.

\section{Monte carlo simulation}


To understand the interaction between the investigated compound and the reinforced steel surface, MC simulation was used to give a clear picture about the adsorption mechanism. Consequently, Fig. 12 shows the side and top views of the most appropriate adsorption configurations for the examined compound protonated and unprotonated on the steel surface obtained by the adsorption locator module. Also, the recorded results using the MC simulation were tabulated in Table 7 such as the total energy of the substrate/adsorbate configuration, the adsorption energy for relaxed adsorbate molecules, rigid adsorption energy for unrelaxed adsorbate molecules, and deformation energy for relaxed adsorbate molecules. Moreover, the adsorption energy is the sum of rigid adsorption energy and deformation energy for the adsorbate molecules. As shown in Table 7, unprotonated has higher adsorption energy compared to protonated which predicts the strong adsorption of unprotonated on the reinforced steel surface forming stable adsorbed layers and protects the steel against dissolution. The $\mathrm{dE}_{\text {ads }} / \mathrm{dNi}$ records the energy of metal-adsorbate configuration where one of the adsorbates has been removed. The $\mathrm{dE}_{\text {ads }} / \mathrm{dNi}$ value for unprotonated $\left(-206.1 \mathrm{kcal} \mathrm{mol}^{-1}\right)$ is greater than protonated molecule $\left(-174.1 \mathrm{kcal} \mathrm{mol}^{-1}\right)$ which indicates a more adsorption of unprotonated molecule than protonated molecule. Furthermore, the $\mathrm{dE}_{\mathrm{ads}} / \mathrm{dNi}$ value for water is small compared with its value for unprotonated and protonated indicating the strong adsorption of examined inhibitor molecules compared to water molecules. Therefore, the unprotonated and protonated molecules are surely adsorbed on the reinforced steel surface and establishing stable adsorbed layers causing a corrosion protection for steel surface in the corrosive medium as confirmed by both experimental and theoretical investigations. From MC data, the order of inhibition for this inhibitor is: Unprotonated > protonated

Table 7. MD calculated parameters most stable configuration of investigated compound on Fe (110) surface in vacuum conditions

\begin{tabular}{|c|c|c|c|c|c|c|}
\hline Structures & $\begin{array}{r}\text { Total } \\
\text { energy }\end{array}$ & $\begin{array}{l}\text { Adsorptio } \\
n \\
\text { energy }\end{array}$ & $\begin{array}{l}\text { Rigid } \\
\text { adsorption } \\
\text { energy }\end{array}$ & $\begin{array}{l}\text { Deformation } \\
\text { energy }\end{array}$ & $\begin{array}{l}\text { Compound } \\
\mathrm{dE}_{\mathrm{ad}} / \mathrm{dNi}\end{array}$ & $\begin{array}{l}\mathrm{H}_{2} \mathrm{O} \\
\mathrm{dE}_{\mathrm{ad}} / \mathrm{dNi}\end{array}$ \\
\hline $\begin{array}{l}\mathrm{Fe}\left(\begin{array}{lll}1 & 1 & 0\end{array}\right) \\
\text { unprotonated } \\
\text { /H2O }\end{array}$ & $\begin{array}{l}- \\
3277.6\end{array}$ & -3197.2 & -3353.5 & 156.2 & -206.1 & -6.5 \\
\hline $\begin{array}{l}\mathrm{Fe}\left(\begin{array}{lll}1 & 1 & 0\end{array}\right) \\
\text { protonated } / \mathrm{H} 2 \mathrm{O}\end{array}$ & $\begin{array}{l}- \\
3243.2\end{array}$ & -3171.2 & -3324.4 & 153.1 & -174.1 & -1.4 \\
\hline
\end{tabular}




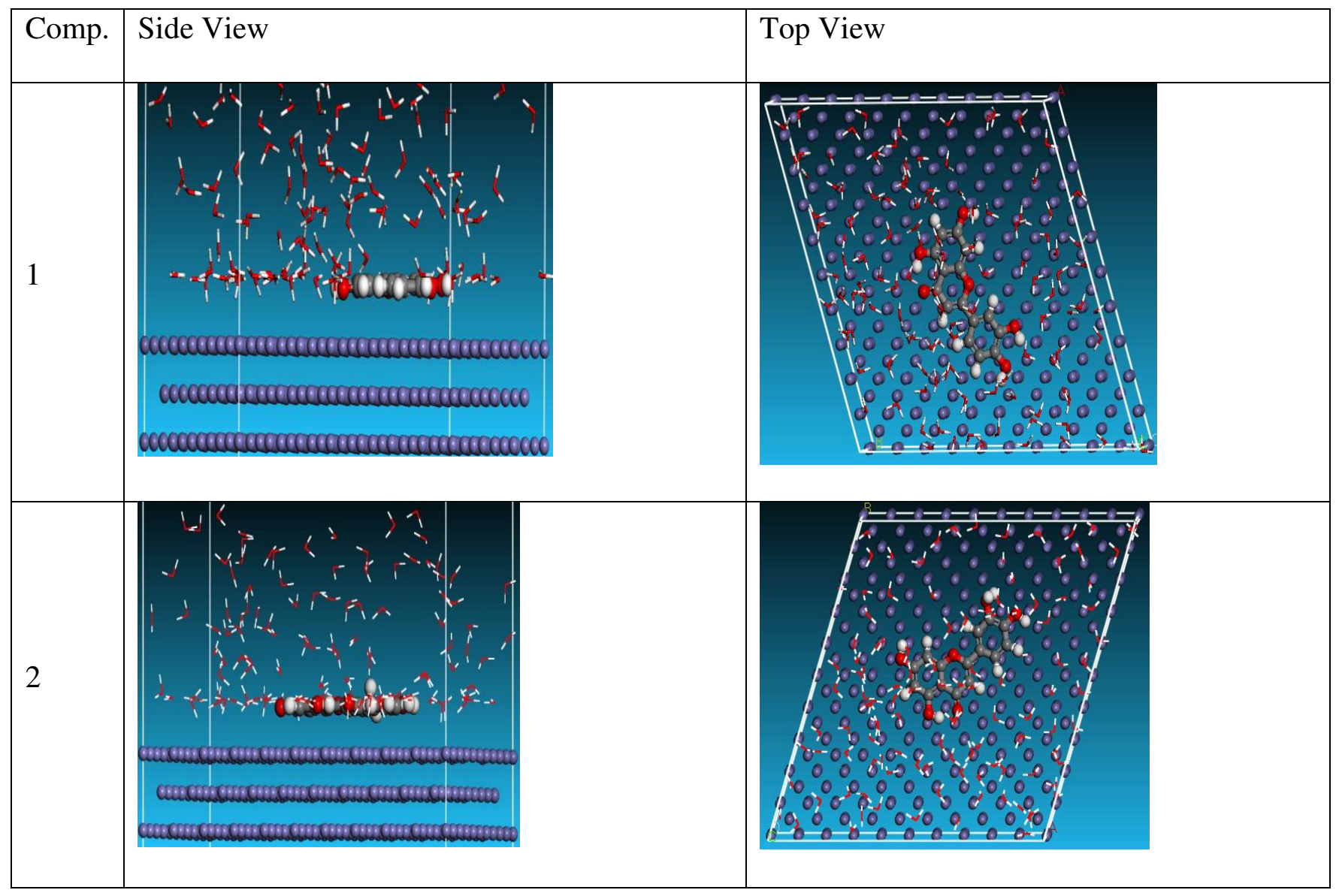

Figure 12. Side and top views for MD simulations with the most stable configuration of investigated compound on Fe (110) surface in vacuum conditions

\section{Corrosion mechanism of aizoon extract}

The role of Aizoon extract on the SS430 corrosion mitigation in $\mathrm{HCl}$ solution is schematically presented in Scheme 1. According to the previous studies, the steel surface can be positively charged in exposure with $\mathrm{HCl}$ solution [34]. Therefore, the chloride ions $(\mathrm{Cl}-)$ adsorbs on the steel surface. In the next step, the protonated Aizoon extract molecules adsorb on the metal substrate through electrostatic adsorption mechanism. As shown in scheme 1, the Aizoon extract molecules like 5,7-dihydroxy chromone, pyrogallol, kaempferol, protocatechuic acid, vallinic acid, thymine, caffeic acid, quercetin, p- hydroxy benzoic acid, Gallic acid, and luteolin include plenty number of oxygen heteroatoms. These heteroatoms could donate their free electrons to the unfilled orbitals of the iron atoms, resulting in the Aizoon extract molecules adsorption on the SS430 surface through chemisorption mechanism [35]. Thus, extract molecules can adsorb on SS430 surface by following ways: I0Electrostatic interaction between the charged molecules and charged metal ii) Interaction of-electrons with the metal iii) Interaction of unshared pair of electrons in the molecule with the metal iv) The combination of all effects [36] 


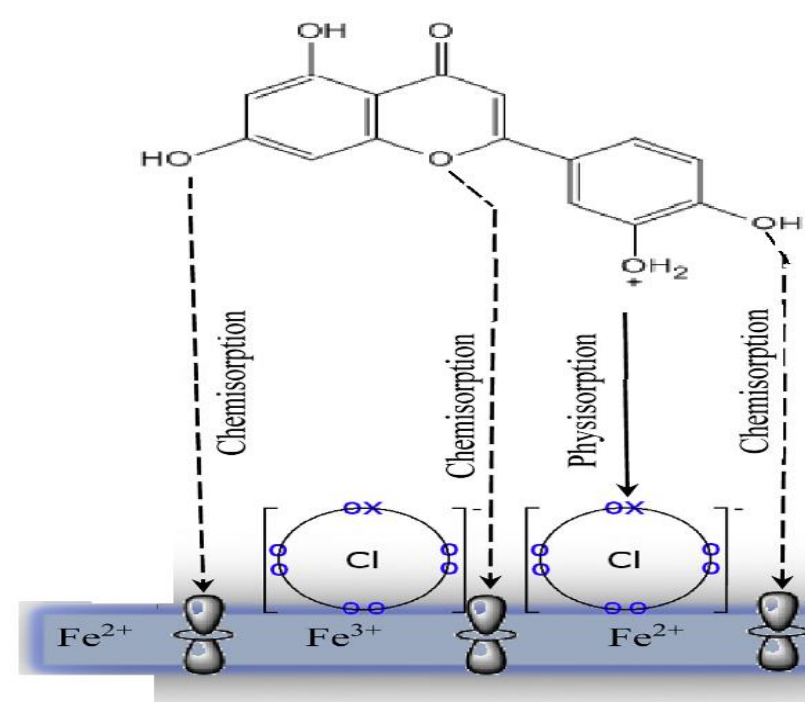

Scheme 1 The schematic illustration of different modes of adsorption by luteolin on SS430 in 1 $\mathrm{M} \mathrm{HCl}$ interface

\section{Conclusions}

Aizoon extract is a good green extract for corrosion of SS430 in 2M HCl. From all experiments it was found that the inhibition efficiency increased with increasing Aizoon extract concentration and decreased by raising the temperature. Thus, the Aizoon extract compounds adsorbed physically on the SS430 surface. Furthermore, it was confirmed that the adsorption of Aizoon compounds on SS430 surface is spontaneous process and obeys the Langmuir adsorption model. The activation energy for the corrosion of SS430 increased significantly with extract compared to that in free acid demonstrating the inhibition characteristic of Aizoon extract. Potentiodynamic polarization measurement suggested that Aizoon extract can be adsorbed on both anodic and cathodic sites. However, the extract inclined to be adsorbed on cathodic sites. Therefore, Aizoon extract can be used as an additive in cathodic protection.

\section{Acknowledgement}

The authors thanks Dr. Ashraf Nofal associate prof of Fungi and Plant Pathology, Environmental Studies and Research Institute, Sustainable Department, University of Sadat City, El-Menoufia, Egypt. Who is the collected Aizoon plant from Sadat city desert, Egypt

\section{Declarations}

Conflict of interest: All Authors declare that there is not any conflict of interest in this study.

A Statements: The corresponding author declare that this study complies with relevant institutional, national, and international guidelines and legislation. 
Permission for collection of plant: Dr. Ashraf Nofal associate prof of Fungi and Plant Pathology, Egypt, University of Sadat City, Environmental Studies and Research, Institute, Sustainable Department. Who is collected the plant from Sadat city desert, Egypt

Data Availability. The data sets generated during and/or analyzed during the current study are available from the corresponding author upon reasonable request.

\section{References}

1. Devi M.K., Selvarani F.R. Adsorption and Corrosion inhibition of Stainless steel in Hydrochloric acid by Sulphanilic acid. Int. J. Adv. Res. \& Technol. 3(8),48-52(20`4).

2. Yang Q., Luo J.L. Effects of hydrogen and tensile stress on the breakdown of passive films on type 304 stainless steel. Electrochim Acta. 46(6),851-859 (2001).

3. Abd El Meguid E.A., Mahmoud N.A., Abd El Rehim S.S. The effect of some sulphur compounds on the pitting corrosion of type 304 stainless steel. Mater Chem Phys. 63(1),67-74 (2000).

4. Ameer M.A., Fekry A.M., Heakal F.E.T. Electrochemical behaviour of passive films on molybdenum-containing austenitic stainless steels in aqueous solutions. Electrochim Acta. 50(1), 43-49 (2004).

5. El-Egamy S.S., Badaway W.A. Passivity and passivity breakdown of 304 stainless steel in alkaline sodium sulphate solutions. J Appl Electrochem. 34(11),1153-1158(2004).

6. Torres V.V., Amado R.S., De Sá C.F., et al Inhibitory action of aqueous coffee ground extracts on the corrosion of carbon steel in $\mathrm{HCl}$ solution. Corros Sci. 53(7), 2385-2392(2011).

7. Sherif S.M., Almajid A.A. Surface protection of copper in aerated 3.5\% sodium chloride solutions by 3-amino-5-mercapto-1, 2, 4-triazole as a copper corrosion inhibitor. J Appl Electrochem.: 40(8), 1555-1562 (2010).

8. Fouda A.S., El-Maksoud S.A., El-Hossiany A., Ibrahim A. Evolution of the corrosion-inhibiting efficiency of novel hydrazine derivatives against corrosion of stainless steel 201 in acidic medium. Int J Electrochem Sci. 14(7), 6045-6064 (2019). doi:10.20964/2019.07.65

9. Fouda A.S., Abdel-Latif E., Helal H.M., El-Hossiany A. Synthesis and Characterization of Some Novel Thiazole Derivatives and Their Applications as Corrosion Inhibitors for Zinc in $1 \mathrm{M}$ Hydrochloric Acid Solution. Russ J Electrochem.57(2), 159-171(2021). doi:10.1134/S1023193521020105

10. Sherif E.S.M., Erasmus R.M., Comins J.D. Corrosion of copper in aerated acidic pickling solutions and its inhibition by 3-amino-1, 2, 4-triazole-5-thiol. J Colloid Interface Sci. 306(1), 96-104 (2007).

11. Motawea M.M., El-Hossiany A., Fouda A.S. Corrosion control of copper in nitric acid solution using chenopodium extract. Int J Electrochem Sci. 14,1372-1387 (2019).

12. Sherif SM, Erasmus RM, Comins JD Effects of 3-amino-1, 2, 4-triazole on the inhibition of copper corrosion in acidic chloride solutions. J Colloid Interface Sci. 311(1):144-151.

13. Sherif S.M., Erasmus R.M., Comins J.D. Inhibition of corrosion processes on copper in aerated sodium chloride solutions by 5-(3-aminophenyl)-tetrazole. J Appl Electrochem. 39(1), 83-91 (2009).

14. Sherif S.M., Erasmus R.M., Comins J.D. Inhibition of copper corrosion in acidic chloride pickling solutions by 5-(3-aminophenyl)-tetrazole as a corrosion inhibitor. Corros Sci. 50(12), 3439-3445 (2008).

15. Qiang Y., Zhang S., Tan B., Chen S. Evaluation of Ginkgo leaf extract as an eco-friendly corrosion inhibitor of X70 steel in $\mathrm{HCl}$ solution. Corros Sci. 133, 6-16 (2018).

16. Fouda A.S., El-Ghaffar M.A.A., Sherif M.H., El-Habab A.T., El-Hossiany A. Novel Anionic 4- 
Tert-Octyl Phenol Ethoxylate Phosphate Surfactant as Corrosion Inhibitor for C-steel in Acidic Media. Prot Met Phys Chem Surfaces. 56(1),189-201 (2020). doi:10.1134/S2070205120010086

17. Suprapto W., Soenoko R., Suprapto A., Gapsari F. The Inhibition of 304ss in Hydrochloric Acid Solution by Cera Alba Extract. J Eng Sci Technol. 12(8), 2078-2090 (2017).

18. Fouda A.S., Azeem M.A., Mohamed S., El-Desouky A. Corrosion inhibition and adsorption behavior of nerium oleander extract on carbon steel in hydrochloric acid solution. Int J Electrochem Sci. 14,3932-3948 (2014).

19. Lin Z., Fang Y., Huang A., Chen L., Guo S., Chen J. Chemical constituents from Sedum aizoon and their hemostatic activity. Pharm Biol. 52(11), 1429-1434 (2014).

20. Zaafarany I., Abdallah M. Ethoxylated fatty amide as corrosion inhibitors for carbon steel in hydrochloric acid solution. Int J Electrochem Sci. 5(1), 18-28 (2010).

21. Zerga B., Attayibat A., Sfaira M., et al Effect of some tripodal bipyrazolic compounds on C38 steel corrosion in hydrochloric acid solution. J Appl Electrochem. 40(9),1575-1582 (2010).

22. Fouda A.S., El-Dossoki F.I., El-Hossiany A., Sello E.A. Adsorption and Anticorrosion Behavior of Expired Meloxicam on Mild Steel in Hydrochloric Acid Solution. Surf Eng Appl Electrochem. 56(4), 491-500 (2020).

23. Amin M.A., Abd El-Rehim S.S., El-Sherbini E.E.F., Bayoumi R.S. The inhibition of low carbon steel corrosion in hydrochloric acid solutions by succinic acid: Part I. Weight loss, polarization, EIS, PZC, EDX and SEM studies. Electrochim Acta. 52(11),3588-3600(2007).

24. Fouda A.S., Abd El-Maksoud S.A., Belal A.A.M., El-Hossiany A., Ibrahium A. Effectiveness of some organic compounds as corrosion inhibitors for stainless steel 201 in $1 \mathrm{M} \mathrm{HCl}$ : experimental and theoretical studies. Int J Electrochem Sci. 13, 9826-9846(2018).

25. Cid M., Petit M.C. Discussion of "Reaction Model for Iron Dissolution Studied by Electrode Impedance I. Experimental Results and Reaction Model"'[M. Keddam, OR Mattos, and H. Takenouti]. J Electrochem Soc. 128(12), 257-266 (1981).

26. Veloz M.A., Gonzalez I. Electrochemical study of carbon steel corrosion in buffered acetic acid solutions with chlorides and $\mathrm{H}_{2} \mathrm{~S}$. Electrochim Acta. 48(2),135-144 (2002).

27. Odewunmi N.A., Umoren S.A., Gasem Z.M. Watermelon waste products as green corrosion inhibitors for mild steel in $\mathrm{HCl}$ solution. J Environ Chem Eng. 3(1),286-296 (2015).

28. Bosch R.W., Hubrecht J., Bogaerts W.F., Syrett B.C. Electrochemical frequency modulation: a new electrochemical technique for online corrosion monitoring. Corrosion. 57(1), 60-70 (2001).

29. Prabhu R.A., Venkatesha T.V., Shanbhag A.V., Kulkarni G.M., Kalkhambkar R.G. Inhibition effects of some Schiff's bases on the corrosion of mild steel in hydrochloric acid solution. Corros Sci. 50(12), 3356-3362 (2008).

30. Morozan A., Goellner V., Nedellec Y., Hannauer J., Jaouen F. Effect of the transition metal on metal-nitrogen-carbon catalysts for the hydrogen evolution reaction. $J$ Electrochem Soc. 162(9):H719-726 (2015).

31. Bichara L.C., Bimbi M.V.F., Gervasi C.A., Alvarez P.E., Brandán S.A. Evidences of the formation of a tin (IV) complex in citric-citrate buffer solution: A study based on voltammetric, FTIR and ab initio calculations. J Mol Struct. 1008, 95-101 (2012).

32. Fouda A.S., Ahmed R.E., El-Hossiany A. Chemical, Electrochemical and Quantum Chemical Studies for Famotidine Drug as a Safe Corrosion Inhibitor for $\alpha$-Brass in $\mathrm{HCl}$ Solution. Prot Met Phys Chem Surfaces. 57(2),398-411 (2021).

33. Khaled M.A., Ismail M.A., El-Hossiany A., Fouda A.S. Novel pyrimidine-bichalcophene derivatives as corrosion inhibitors for copper in $1 \mathrm{M}$ nitric acid solution. $R S C A d v$. 11(41):25314- 
25333 (2021). doi:10.1039/d1ra03603c

34. Zhang K., Yang W., Yin X., et al Amino acids modified konjac glucomannan as green corrosion inhibitors for mild steel in HCl solution. Carbohydr Polym. 181, 191-199 (2018).

35. Singh A.K. Inhibition of mild steel corrosion in hydrochloric acid solution by 3-(4-((Z)-indolin-3ylideneamino) phenylimino) indolin-2-one. Ind Eng Chem Res. 51(8),3215-3223 (2012).

36. Singh A.K., Quraishi M.A. Effect of Cefazolin on the corrosion of mild steel in $\mathrm{HCl}$ solution. Corros Sci. 52(1),152-160 (2010). doi:10.1016/j.corsci.2009.08.050 